\title{
The Rational Approximation of Functions which Are Formally Defined by a Power Series Expansion
}

\author{
By P. Wynn
}

1. Introduction. The advent of high speed digital computers and the consequent intensification of interest in the study of numerical analysis has caused considerable attention to be paid to the problem of obtaining approximation formulas for functions which occur in the theory of mathematical physics. It is the purpose of this note to describe the theory underlying various methods of obtaining rational approximations to functions which are formally defined by a power series expansion; it is assumed that the power series concerned are quite general in character, and that the functions with which they are associated do not satisfy a particular functional equation which would permit the use of any special method. The theory is then subjected to a detailed analysis in terms of the computational steps involved, and a comparison, with regard to computational efficiency, of the rarious methods which may be devised for obtaining rational approximations is given.

2. The Pade Table and the $E$-Array. The approximation to the function defined by the power series

$$
\beta(x)=c_{0}+c_{1} x+c_{2} x^{2}+\cdots
$$

by a rational function of the form

$$
\frac{l_{\mu, \nu}(x)}{V_{\mu, \nu}(x)}=\frac{\alpha_{\mu, 0}+\alpha_{\mu, 1} x+\cdots+\alpha_{\mu, \nu} x^{\nu}}{\beta_{0, \nu}+\beta_{1, \nu} x+\cdots+\beta_{\mu, \nu} x^{\mu \mu}}
$$

can be systematized [1], [2, p. 420], [3, 377] by imposing the condition, where this is possible, that the expansion of (2.2) in ascending powers of $x$ should agree with the power series $(2.1)$ as far as the term in $x^{\mu+\nu}$, i.e., that the power series expansions of $\beta(x) V_{\mu, \nu}(x)=C_{\mu, \nu}(x)$ should commence with a term in $x^{\mu+\nu+1}$. This leads immediately to the set of linear equations.

$$
\begin{aligned}
& c_{0} \beta_{0, \nu}=\alpha_{\mu, \nu} \\
& c_{1} \beta_{0, \nu}+c_{1} \beta_{1, \nu}=\alpha_{\mu, 1} \\
& \cdots \cdots \cdots \cdots \cdots \cdots \cdots \\
& c_{\nu} \beta_{0, \nu}+c_{\nu-1} \beta_{1, \nu}+\cdots+c_{\nu-\mu} \beta_{\mu, \nu}=\alpha_{\mu, \nu} \\
& c_{\nu+1} \beta_{0, \nu}+c_{\nu} \beta_{1, \nu}+\cdots+c_{\nu-\mu+1} \beta_{\mu, \nu}=0 \\
& \cdots \cdots \cdots \cdots \cdots \cdots \cdots \cdots \cdots \\
& c_{\nu+\mu} \beta_{0, \nu}+c_{\nu+\mu-1} \beta_{1, \nu}+\cdots+c_{\nu} \beta_{\mu, \nu}=0
\end{aligned}
$$

which serves uniquely to define the coefficients in $(2.2)$ if the restriction $\beta_{0, \nu}=1$ is imposed. The rational functions (2.2), determined by equations (2.3), may be placed in an array in which the quotient $U_{\mu, \nu}(x) / V_{\mu, \nu}(x)$ is at the intersection of

Received November 5, 1959. 
the $(\nu+1)$ th column and the $(\mu+1)$ th row. Such an array is referred to as the Padé table of the function $\beta(x)$, and the condition that it may be constructed from the systems of equations (2.3) is that all the Hankel determinants

$$
H_{m}{ }^{(n)}=\left|\begin{array}{lccc}
c_{m} & c_{m+1} & \cdots & c_{m+n-1} \\
c_{m+1} & c_{m+2} & \cdots & c_{m+n} \\
: & : & & : \\
c_{m+n-1} & c_{m+n} & \cdots & c_{m+2 n-2}
\end{array}\right|
$$

should be non-zero.

Since the successive convergents of the continued fraction

$$
\frac{c_{m}}{1-} \frac{q_{1}{ }^{(m)} x}{1-} \frac{e_{1}{ }^{(m)} x}{1-} \cdots \frac{q_{r}{ }^{(m)} x}{1-} \frac{e_{r}{ }^{(m)} x}{1-} \cdots
$$

are rational functions of $x$, it is to be expected that there is a connection between the theory of continued fractions and the Pade table. In fact, if (2.5) is the continued fraction expansion, which may be derived by a number of methods one of which will explicitly be described in a later section, of the power series

$$
\sum_{r=0}^{\infty} c_{m+r} x^{r}
$$

then $[2$, p. 447] the quotients

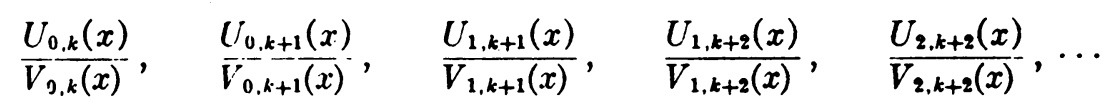

are the successive convergents of the continued fraction

$$
c_{0}+c_{1} x+\cdots+c_{k} x^{k}+\frac{c_{k+1} x^{k+1}}{1-} \frac{q_{1}{ }^{(k+1)} x}{1-} \frac{e_{1}{ }^{(k+1)} x}{1-} \frac{q_{2}{ }^{(k+1)} x}{1-} \frac{e_{2}{ }^{(k+1)} x}{1-} \cdots
$$

while the quotients

$$
\frac{U_{k, 0}(x)}{V_{k, 0}(x)}, \quad \frac{U_{k, 1}(x)}{\Gamma_{k, 1}(x)}, \quad \frac{U_{k+1,1}(x)}{V_{k+1,1}(x)}, \quad \frac{U_{k+1,2}(x)}{V_{k+1,2}(x)}, \quad \frac{U_{k+2,2}(x)}{V_{k+2,2}(x)}, \cdots
$$

are the successive convergents of the continued fraction

$$
\frac{1}{d_{0}+d_{1} x+\cdots+d_{k} x^{k}+} \frac{d_{k+1} x^{k+1}}{1-} \frac{q_{1}^{(k+1) \prime} x}{1-} \frac{e_{1}^{(k+1) \prime} x}{1-} \frac{q_{2}^{(k+1) \prime} x}{1-} \frac{e_{2}{ }^{(k+1) \prime} x}{1-} \cdots
$$

where, formally

$$
\sum_{r=0}^{\infty} d_{k+r} x^{r}=\frac{d_{k}}{1-} \frac{q_{1}{ }^{(k) \prime} x}{1-} \frac{e_{1}{ }^{(k) \prime} x}{1-} \frac{q_{2}{ }^{(k) \prime} x}{1-} \frac{e_{2}{ }^{(k) \prime} x}{1-} \cdots
$$

and

$$
\left(\sum_{r=0}^{\infty} c_{r} x^{r}\right)\left(\sum_{r=0}^{\infty} d_{r} x^{r}\right)=1
$$

Before proceeding further it will be stated that rational approximations will be derived which relate to the series 


$$
\beta(x)=\sum_{r=0}^{\infty} c_{r} x^{r}
$$

and others which relate to the series

$$
F(z)=\sum_{r=0}^{\infty} c_{r} z^{-r-1}
$$

Since these series may be transformed into each other by means of the simple relationships

$$
\begin{aligned}
& \beta(x)=x^{-1} F\left(x^{-1}\right) \\
& F(z)=z^{-1} \beta\left(z^{-1}\right),
\end{aligned}
$$

it seems to be most economical to refer all the working to one of the series and further, it would seem to be a cause for surprise that this should be a matter for discussion. However, if the series (2.14) is rewritten as a power series in $x=z^{-1}$ it will be observed that the constant term is missing, i.e., that the condition that all the Hankel determinants (2.4) should be non-zero is violated. If on the other hand the series (2.13) is treated with methods to be described, there results such a wilful perversion of certain classical results, such as occur, for example, in the theory of orthogonal polynomials, as would encourage some other course of action to be adopted. A yet more decisive reason for difficulty is contained in the observation that the Padé table is essentially a symmetric structure in the sense that the power series expansion of $\{\beta(x)\}^{-1}$ has the same form as that of $\beta(x)$, whereas the power series expansion of $\{F(z)\}^{-1}$ has not the same form as that of $F(z)$.

Accordingly, the rational expressions $E_{n}{ }^{(m)}(z)$ of the form

$$
E_{n}{ }^{(m)}(z)=\frac{r_{n}{ }^{(m)}(z)}{{p_{n}{ }^{(m)}(z)}{ }^{(m)}}
$$

where $p_{n}{ }^{(m)}(z)$ is the polynomial of the $n$th degree

$$
p_{n}^{(m)}(z)=\sum_{s=0}^{n} k_{n, s}^{(m)} z^{s},
$$

$r_{n}^{(m)}(z)$ is a function of the form

$$
r_{n}{ }^{(m)}(z)=z^{-m} \sum_{s=0}^{m+n-1} i_{n, s}^{(m)} z^{s}, \quad r_{0}^{\left({ }^{())}\right.}(z)=0
$$

and the series expansion of $E_{n}{ }^{(m)}(z)$ in inverse powers of $z$ agree with that of $F(z)$ as far as the term containing $z^{-m-2 n}$, will be arranged in the following array:

$$
\begin{aligned}
& E_{0}^{(0)}(z) \\
& E_{0}{ }^{(1)}(z) \quad E_{1}{ }^{(0)}(z) \\
& E_{0}{ }^{(2)}(z) \quad E_{1}{ }^{(1)}(z) \quad E_{2}{ }^{(0)}(z) \\
& E_{0}{ }^{(3)}(z) \quad E_{1}{ }^{(2)}(z) \quad E_{2}{ }^{(1)}(z) \quad E_{3}{ }^{(0)}(z) \\
& \vdots \quad \vdots \quad \vdots \quad \vdots \quad \vdots \\
& E_{0}{ }^{(m)}(z) \quad E_{1}{ }^{(m-1)}(z) \quad E_{2}{ }^{(m-2)}(z) \quad E^{(m-3)}(z) \cdots E_{m}{ }^{(0)}(z)
\end{aligned}
$$


in which the quantity $E_{n}{ }^{(m)}(z)$ occurs at the intersection of the $(n+1)$ th column and the $(m+1)$ th diagonal. This array will be referred to throughout this paper as the $E$-array. Clearly the constituents ${p_{n}}^{(m)}(z), r_{n}{ }^{(m)}(z)$ of the function $E_{n}{ }^{(m)}(z)$ may also be displayed in the same array. If, in the notation of equation (2.18), the restriction

$$
k_{n, n}^{(m)}=1
$$

is imposed, it is clear that

$$
z^{-n} p_{n}^{(m)}(z)=V_{n, m+n-1}(x), z x=1
$$

and that

$$
z^{-n+1} r_{n}{ }^{(m)}(z)=U_{n, m+n-1}(x), z x=1 .
$$

If, as is convenient from the point of view of numerical computation, the Pade table is regarded as an array of vector pairs

$$
\left(\alpha_{\mu, 0}, \alpha_{\mu, 1}, \cdots, \alpha_{\mu, \nu}\right), \quad\left(\beta_{0, \nu}, \beta_{1, \nu}, \cdots, \beta_{\mu, \nu}\right),
$$

so that

$$
U_{\mu, \nu}(x)=\left(\alpha_{\mu, 0}, \alpha_{\mu, 1}, \cdots, \alpha_{\mu, p}\right)\left(1, x, \cdots, x^{\prime}\right)^{T}
$$

and

$$
V_{\mu, \nu}(x)=\left(\beta_{0, \nu}, \beta_{1, \nu}, \cdots, \beta_{\mu, \nu}\right)\left(1, x, \cdots, x^{\mu}\right)^{T},
$$

and the $E$-array is regarded as an array of vector pairs

$$
\left(k_{n, 0}^{(m)}, k_{n, 1}^{(m)}, \cdots, k_{n, n}^{(m)}\right), \quad\left(i_{n, 0}^{(m)}, i_{n, 1}^{(m)}, \cdots, i_{m+n-1}^{(m)}\right)
$$

so that

$$
p_{n}^{(m)}(z)=\left(k_{n, 0}^{(m)}, k_{n, 1}^{(m)}, \cdots, k_{n, n}^{(m)}\right)\left(1, z, \cdots, z^{n}\right)^{T}
$$

and

$$
r_{n}^{(m)}(z)=z^{-m}\left(i_{n, 0}^{(m)}, i_{n, 1}^{(m)}, \cdots, i_{n, m+n-1}^{(m)}\right)\left(1, z, \cdots, z^{m+n+1}\right)^{T}
$$

it is seen that the whole $E$-array may be obtained from the Pade table by transposing the Padé table, deleting these terms lying above the diagonal beginning with the second term of the first row, and placing the quantity $E_{0}{ }^{(0)}(z)=0$ at the peak of the array; conversely, part of the Padé table may be obtained from the $E$-array by removing the entry $E_{0}{ }^{(0)}(z)$ and transposing the $E$-array about the diagonal $E_{n}{ }^{(1)}(z), n=0,1, \cdots$.

Approximations relating to both series (2.13) and (2.14) will be derived, the series chosen for treatment in a particular case being selected according to the dictates of convenience. The variable used will indicate the series under consideration. Methods for deriving rational approximation formulas will now be discussed.

\section{The E-Array.}

\subsection{Orthogonal Polynomials}

The various questions associated with the central problem of obtaining rational functions from power series expansions are unified and greatly clarified by an appeal 
to the classical theory of orthc $\sigma$ nal polynomials. This is usually introduced [4, p. 153] by focusing attention upon the integral formula

$$
\int_{a}^{b} p_{r}(t) p_{s}(t) d \phi(t)= \begin{cases}0 & r \neq s \\ \omega_{s} & r=s\end{cases}
$$

which serves uniquely to define a sequence of polynomials $p_{r}(t) \quad r=0,1, \cdots$, and it is then noted at some later stage that if

$$
\int_{a}^{b} t^{s} d \phi(t)=c_{s}
$$

then formally

$$
\int_{a}^{b} \frac{1}{z-t} d \phi(t)=\sum_{r=0}^{\infty} c_{r} z^{-r-1}
$$

Since, however, the moments $c_{s}$ are the central feature of the problem in hand, and the introduction of the integral and weight function $\phi(t)$ ( in view of the difficulty of envisaging an appropriate form for $\phi(t))$ may even serve as an impediment to understanding, the present treatment will slightly be varied.

Accordingly, define a process $I$ by

$$
I\left(t^{s}\right)=c_{s} \quad s=0,1, \cdots
$$

and construct a sequence of orthogonal polynomials $p_{n}{ }^{(m)}(z) n=0,1, \cdots, m=0$, $1, \cdots$, from the relations

$$
I\left(t^{m+8}{p_{n}}^{(m)}(t)\right)=\left\{\begin{array}{lr}
0 & 0 \leqq s \leqq n-1 \\
\omega_{n} & s=n
\end{array}\right.
$$

where $\omega_{n}$ is so chosen that in the representation

$$
\begin{aligned}
p_{n}{ }^{(m)}(z) & =\sum_{s=0}^{n} k_{n, s}^{(m)} z^{s} \\
k_{n, n}^{(m)} & =1
\end{aligned}
$$

Construct a second sequence of polynomials

$$
o_{n}{ }^{(m)}(z)=\sum_{s=0}^{n-1} j_{n, s}^{(m)} z^{*}
$$

from the relation

$$
o_{n}{ }^{(m)}(z)=I\left\{\frac{t^{m}\left(p_{n}{ }^{(m)}(z)-p_{n}{ }^{(m)}(t)\right)}{z-t}\right\} \quad n=1, z, \cdots ; m=0,1, \cdots
$$

These are the classical associated polynomials occurring in the theory of orthogonal polynomials [4, p. 162].

Note in passing that formally

$$
I\left\{\frac{t^{m}}{z-t}\right\}=\sum_{s=0}^{\infty} c_{m+s} z^{-s-1} .
$$

Consider the quotient $o_{n}{ }^{(m)}(z) / p_{n}{ }^{(m)}(z)$, there follows 


$$
\begin{aligned}
\frac{o_{n}{ }^{(m)}(z)}{p_{n}{ }^{(m)}(z)} & =I\left\{\frac{t^{m}\left(p_{n}{ }^{(m)}(z)-p_{n}{ }^{(m)}(t)\right)}{z-t}\right\} / p_{n}{ }^{(m)}(z) \\
& =I\left\{\frac{t^{m}}{z-t}\right\}-\frac{1}{p_{n}^{(m)}(z)} I\left\{\frac{t^{m} p_{n}{ }^{(m)}(t)}{z-t}\right\} \\
& =\sum_{s=0}^{\infty} c_{m+s} z^{-s-1}-\left\{z^{-n}-k_{n, n-1}^{(m)} z^{-n-1}+\cdots\right\} I\left\{\sum_{s=0}^{\infty} p_{n}{ }^{(m)}(t) t^{m+s} z^{-s-1}\right\} \\
& =\sum_{n=0}^{\infty} c_{m+s} z^{-s-1}+P_{2 n+1}(z)
\end{aligned}
$$

where $P_{2 n+1}(z)$ is a power series beginning with a term in $z^{-2 n-1}$. Define a third sequence of functions $r_{n}^{(m)}(z)$, where

$$
z^{m} r_{n}^{(m)}(z)=\sum_{s=0}^{m+n-1} i_{n, 8}^{(m)} z^{s}
$$

by means of the relation

$$
r_{n}^{(m)}(z)=I\left\{\frac{z^{m} p_{n}^{(m)}(z)-t^{m} p_{n}^{(m)}(t)}{z-t}\right\},
$$

and note that

$$
\frac{r_{n}{ }^{(m)}(z)}{p_{n}{ }^{(m)}(z)}=\sum_{n=0}^{m-1} c_{s} z^{-s-1}+z^{-m} \frac{o_{n}{ }^{(m)}(z)}{p_{n}{ }^{(m)}(z)}=\sum_{s=0}^{\infty} c_{s} z^{-s-1}+P_{m+2 n+1}(z)
$$

Thus it can be seen that the construction of the $E$-array may be greatly facilitated if the functions $o_{n}{ }^{(m)}(z), p_{n}{ }^{(m)}(z)$ and $r_{n}{ }^{(m)}(z)$ may be constructed with moderate ease. Results which enable this to be done, and others which will be of later interest, are now given.

Eliminating the coefficients $k_{n, s}^{(m)} \quad s=0,1, \cdots, n$, between $n$ equations of the form (3.1.5) with $s=0,1, \cdots, n-1,(3.1 .6)$ amd (3.1.7) there follows the determinantal formula $[4$, p. 158$]$ for the polynomial $p_{n}{ }^{(m)}(z)$

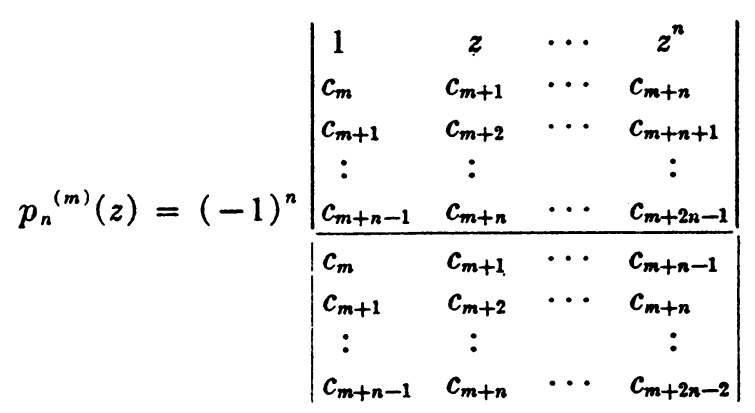

Further it may be shown $[4$, p. 158] that the polynomials satisfy a recursion of the form

$$
\begin{gathered}
p_{r+1}^{(m)}(z)=\left(z-\alpha_{r}^{(m)}\right) p_{r}{ }^{(m)}(z)-\beta_{r-1}^{(m)} p_{r-1}^{(m)}(z) \\
p_{0}{ }^{(m)}(z)=1, \quad p_{1}{ }^{(m)}(z)=z-\alpha_{0}{ }^{(m)},
\end{gathered}
$$

and, from the definitions (3.1.9) and (3.1.13) 


$$
\begin{gathered}
o_{r+1}^{(m)}(z)=\left(z-\alpha_{r}{ }^{(m)}\right) O_{r}{ }^{(m)}(z)-\beta_{r-1}^{(m)} o_{r-1}^{(m)}(z) \\
o_{0}{ }^{(m)}(z)=0, \quad o_{1}{ }^{(m)}(z)=c_{m}
\end{gathered}
$$

and

$$
\begin{gathered}
r_{r+1}^{(m)}(z)=\left(z-\alpha_{r}{ }^{(m)}\right) r_{r}{ }^{(m)}(z)-\beta_{r-1}^{(m)} r_{r-1}^{(m)}(z) \\
r_{0}{ }^{(m)}(z)=\sum_{j=0}^{m-1} c_{s} z^{-s-1}, \quad r_{1}^{(m)}(z)=p_{1}^{(m)}(z) r_{0}^{(m)}(z)+c_{m} z^{-m} .
\end{gathered}
$$

These recursions, in conjunction with equations (3.1.11) and (3.1.14) serve to identify the quotient $o_{n}{ }^{(m)}(z) / p_{n}{ }^{(m)}(z)$ as the $n$th convergent of the continued fraction

$$
F_{m}(z)=\frac{c_{m}}{z-\alpha_{0}{ }^{(m)}-} \frac{\beta_{0}{ }^{(m)}}{z-\alpha_{1}{ }^{(m)}-} \cdots \frac{\beta_{r-1}^{(m)}}{z-\alpha_{r}^{(m)}} \cdots
$$

where formally

$$
F_{m}(z)=\sum_{r=0}^{\infty} c_{m+r} z^{-r-1}
$$

and the quotient $r_{n}{ }^{(m)}(z) / p_{n}{ }^{(m)}(z)$ as the $n$th convergent of the continued fraction

$$
\begin{aligned}
F(z)=\sum_{s=0}^{m-1} c_{s} z^{-s-1} & \\
& +z^{-m}\left\{\frac{c_{m}}{z-\alpha_{0}{ }^{(m)}-}-\frac{\beta_{0}{ }^{(m)}}{z-\alpha_{1}^{(m)}}-\cdots-\frac{\beta_{r-1}^{(m)}}{z-\alpha_{r}^{(m)}-} \cdots\right\}
\end{aligned}
$$

Multiplying equation (3.1.16) through by $t^{m+r-1}$ and replacing $z$ by $t$, it follows, by virtue of equation (3.12), that

$$
I\left(t^{m+r} p_{r}^{(m)}(t)\right)=\beta_{r-1}^{(m)} I\left(t^{m+r-1} p_{r-1}^{(m)}(t)\right)=c_{m} \beta_{0}{ }^{(m)} \beta_{1}{ }^{(m)} \cdots \beta_{r-1}^{(m)}
$$

Similarly, by multiplying the same equation through by $t^{m+r}$, it follows that

$$
I\left(t^{m+r+1} p_{r}{ }^{(m)}(t)\right)=c_{m} \beta_{0}{ }^{(m)} \beta_{1}{ }^{(m)} \cdots \beta_{r-1}^{(m)}\left(\alpha_{0}{ }^{(m)}+\alpha_{1}{ }^{(m)}+\cdots+\alpha_{r}{ }^{(m)}\right)
$$

Equations (3.1.16), (3.1.22), and (3.1.23), taken in conjunction are now sufficient to determine in succession the quantities $k_{r, s}^{(m)}, \beta_{r-1}^{(m)}, \alpha_{r}{ }^{(m)}$ for any preassigned value of $m\left[3\right.$, p. 196]. The coefficients $i_{r . s}^{(m)}$ may be recovered by means of the recursion (3.1.18), or from the condition (3.1.14), or from the recursion (3.1.17) in conjunction with the condition

$$
r_{n}^{(m)}(z)=p_{n}{ }^{(m)}(z) \sum_{s=0}^{m-1} c_{s} z^{-s-1}+z^{-m} o_{n}^{(m)}(z) .
$$

3.2 Stieltjes' Algorithm [5]; [2, p. 326]; [3, p. 20:3].

An algorithm for obtaining the quantities $\alpha_{r}{ }^{(m)}, \beta_{r-1}^{(m)}$ and hence, by virtue of equations (3.1.16) and (3.1.18), the quotients $r_{n}{ }^{(m)}(z) / p_{n}{ }^{(m)}(z)$, is implicit in the followng theorem due to Stieltjes: If the bilinear form $\sum_{p, q-0}^{\infty} c_{m+p+q} x_{p} y_{q}$ is decomposed into

$$
\begin{aligned}
& \sum_{p, q=0}^{\infty} c_{m+p+q} x_{p} y_{q}=c_{m}\left(h_{0,0}^{(m)} x_{0}+h_{1,0}^{(m)} x_{1}+\cdots\right)\left(h_{0,0}^{(m)} y_{0}+h_{1,0}^{(m)} y_{1}+\cdots\right) \\
& +c_{m} \beta_{0}^{(m)}\left(h_{1,1}^{(m)} x_{1}+h_{2,1}^{(m)} x_{2}+\cdots\right)\left(h_{1,1}^{(m)} y_{1}+h_{2,1}^{(m)} y_{2}+\cdots\right) \\
& \quad+c_{m} \beta_{0}{ }^{(m)} \beta_{1}^{(m)}\left(h_{2,2}^{(m)} x_{2}+h_{3,2}^{(m)} x_{3}+\cdots\right)\left(h_{2,2}^{(m)} y_{2}+h_{3,2}^{(m)} y_{3}+\cdots\right)+\cdots
\end{aligned}
$$


then the quantities $h_{r, 8}^{(m)}$ satisfy recursions involving quantities $\alpha_{r}^{(m)}, \beta_{r-1}^{(m)}$ expressible by the matrix equation

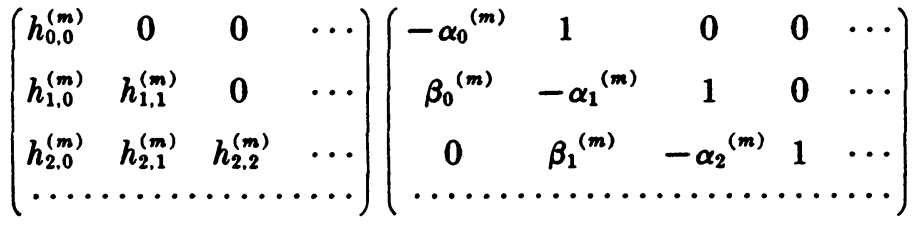

$$
\begin{aligned}
& =\left(\begin{array}{cccc}
h_{1,0}^{(m)} & h_{1,1}^{(m)} & 0 & \cdots \\
h_{2,0}^{(m)} & h_{2,1}^{(m)} & h_{2,2}^{(m)} & \cdots \\
h_{3,0}^{(m)} & h_{3,1}^{(m)} & h_{3,2}^{(m)} & \cdots \\
\cdots \cdots & \cdots & \cdots & \cdots
\end{array}\right)
\end{aligned}
$$

in which

$$
h_{r, r}^{(m)}=1 \quad r=0,1, \cdots,
$$

urthermore, by equating the coefficients of $x_{p} y_{q}$ on the right and left hand sides of equation (3.2.1)

$$
c_{m+p+q}=c_{m} h_{p, 0}^{(m)} h_{l, 1)}^{(m)}+c_{m} \beta_{0}{ }^{(m)} h_{p, 1}^{(m)} h_{q, 1}^{(m)}+c_{m} \beta_{0}{ }^{(m)} \beta_{1}{ }^{(m)} h_{p, 2}^{(m)} h_{q, 2}^{(m)}+\cdots
$$

the series in (3.2.4) being terminated by observing that $h_{r, s}^{(m)}=0$ if $s>r$. Finally, from exuation (3.2.2)

$$
\alpha_{01}^{(m)}=-h_{1,0}^{(m)} \quad \alpha_{r}^{(m)}=h_{r, r-1}^{(m)}-h_{r+1, r}^{(m)} \quad r=1,2, \cdots
$$

The signific:ance of this result is revealed by denoting the triangular matrix in equation (3.2.2) by $\mathbf{H}^{(\text {'m) }}$, the matrix

$$
\left(\begin{array}{llll}
c_{m} & c_{m+1} & c_{m+2} & \cdots \\
c_{m+1} & c_{m+2} & c_{m+3} & \cdots \\
c_{m+2} & c_{m+3} & c_{m+4} & \cdots \\
\cdots & \ldots & \ldots & \ldots
\end{array}\right)
$$

by $\mathbf{C}^{(m)}$, the diagonal matrix

$$
\left(\begin{array}{cccc}
c_{m} & 0 & 0 & \cdots \\
0 & c_{m} \beta_{0}{ }^{(m)} & 0 & \ldots \\
0 & 0 & c_{m} \beta_{0}{ }^{\left({ }^{m}\right)} \beta_{1}{ }^{(n)} & \ldots \\
\ldots \ldots & \ldots \ldots \ldots \ldots \ldots \ldots \ldots \ldots
\end{array}\right)
$$

by $\mathbf{D}^{(m)}$, the vector $\left(x_{1}, x_{1}, x_{2}, \cdots\right)$ by $\mathbf{x}$, the vector $\left(y_{0}, y_{1}, y_{2}, \cdots\right)^{\boldsymbol{T}}$ by $\mathbf{y}$, and rewriting equation (3.2.1) as

$$
\mathrm{xC}^{(m)} \mathrm{y}=\boldsymbol{x} \mathrm{H}^{(m)} \mathbf{D}^{(m)} \mathbf{H}^{(m)} \mathbf{y} .
$$

Thus it is seen immediately that the process of determining the triangular array $\mathbf{H}^{(m)}$ is equivalent to the familiar Gauss-Banachiewicz decomposition for solving linear systems, in which the matrix $D^{(m)}$ is so chosen that the diagonal elements of the matrix $\mathrm{H}^{(m)}$ are unity (see the remark contained upon page 278 of [6]). The relationship between the matrix $\mathbf{H}^{(m)}$ and the matrix 


$$
\mathbf{K}^{(m)}=\left(\begin{array}{cccc}
k_{0.0}^{(m)} & 0 & 0 & \cdots \\
k_{1.0}^{(m)} & k_{1.1}^{(m)} & 0 & \cdots \\
k_{2.0}^{(m)} & k_{2.1}^{(m)} & k_{2.2}^{(n)} & \ldots \\
\cdots & \cdots & \cdots & \cdots
\end{array}\right)
$$

of coefficients in the orthogonal polynomials $p_{n}{ }^{(m)}(z) \quad n=0,1, \cdots$ is revealed by noting that a consequence of equations (3.1.7) and (3.1.22) taken in conjunction is that

$$
I\left(t^{m} p_{r}{ }^{(m)}(t) p_{t}{ }^{(m)}(t)\right)=\left\{\begin{array}{lr}
0 & r=0,1, \cdots, s-1, \\
c_{m} \beta_{0}{ }^{(m)} \beta_{1}{ }^{(m)} \cdots \beta_{r-1}^{(m)} & r=s
\end{array}\right.
$$

or in matrix notation

$$
\mathbf{K}^{(m)} \mathbf{C}^{(m)} \mathbf{K}^{(m) T}=\mathbf{D}^{(m)}
$$

Using the result (3.2.8) it follows that

$$
\mathbf{K}^{(m)} \mathbf{H}^{(m)} \mathbf{D}^{(m)} \mathbf{H}^{(m) \tau} \mathbf{K}^{(m) \tau}=\mathbf{D}^{(m)}
$$

or

$$
\mathbf{K}^{(m)}=\mathbf{H}^{(m)-1} .
$$

The author is indebted to Professors F. L. Bauer and H. Rutishauser for remarks upon this section.

\subsection{The Quotient Difference Algorithm}

If the substitutions

with

$$
\alpha_{s}{ }^{(m)}=q_{s+1}^{(m)}+e_{s}{ }^{(m)} \quad \beta_{s}{ }^{(m)}=e_{s+1}^{(m)} q_{s+1}^{(m)} \quad s=0,1, \ldots
$$

$$
e_{0}^{(m)}=0
$$

are made, the continued fraction (3.1.19) becomes

$$
\frac{c_{m}}{z-q_{1}{ }^{(m)}}-\frac{e_{1}{ }^{(m)} q_{1}{ }^{(m)}}{z-q_{2}{ }^{(m)}-e_{1}{ }^{(m)}-} \cdots \frac{e_{r}{ }^{(m)} q_{r}{ }^{(m)}}{z-q_{r+1}^{(m)}-e_{r}{ }^{(m)}-} \cdots
$$

and may be recognized as the even part [2, p. 197], [3, p. 20],

$$
\frac{c_{m}}{z-} \frac{q_{1}^{(m)}}{1-} \frac{e_{1}^{(m)}}{z-} \frac{q_{2}^{(m)}}{1-}-\frac{e_{2}^{(m)}}{z-} \cdots .
$$

The odd part of (3.3.3) is

$$
\frac{c_{m}}{z}\left\{1+\frac{q_{1}{ }^{(m)}}{z-q_{1}^{(m)}-e_{1}{ }^{(m)}-}-\frac{e_{1}{ }^{(m)} q_{2}{ }^{(m)}}{z-q_{2}{ }^{(m)}-e_{2}{ }^{(m)}}-\frac{e_{r}^{(m)} q_{r+1}^{(m)}}{z-q_{r+1}^{(m)}-e_{r+1}^{(m)}} \cdots\right\} .
$$

However

$$
\sum_{r=0}^{\infty} c_{m+r} z^{-r-1}=\frac{c_{m}}{z}+z^{-1} \sum_{r=0}^{\infty} c_{m+r+1} z^{-r-1}
$$


and hence

$$
\begin{aligned}
& \sum_{r=0}^{\infty} c_{m+r+1} z^{-r-1} \\
& =\frac{c_{m} q_{1}{ }^{(m)}}{z-q_{1}{ }^{(m)}-e_{1}{ }^{(m)}-}-\frac{e_{1}^{(m)} q_{2}{ }^{(m)}}{z-q_{2}{ }^{(m)}-e_{2}{ }^{(m)}-}-\cdots \frac{e_{r}^{(m)} q_{r+1}^{(m)}}{z-q_{r+1}^{(m)}-e_{r+1}^{(m)}-} \cdots
\end{aligned}
$$

Substituting $m+1$ for $m$ in (3.3.2) and equating the coefficients in the resulting continued fraction with those of (3.3.6) there follows the two systems of equations

$$
\begin{gathered}
q_{r}^{(m)}+e_{r}^{(m)}=q_{r}^{(m+1)}+e_{r-1}^{(m+1)} \\
q_{r+1}^{(m)} e_{r}^{(m)}=q_{r}^{(m+1)} e_{r}^{(m+1)}
\end{gathered}
$$

which may progressively be used to derive the coefficients in the continued fraction (3.3.2) from those of the infinite series $\sum_{r=0}^{\infty} c_{m+r} z^{-r-1}$

The quantities $q_{r}{ }^{(m)}, e_{r}{ }^{(m)}$ may be arranged in the following scheme:

$$
q_{1}^{(0)}
$$

$$
e_{0}^{(1)} \quad e_{1}^{(0)}
$$

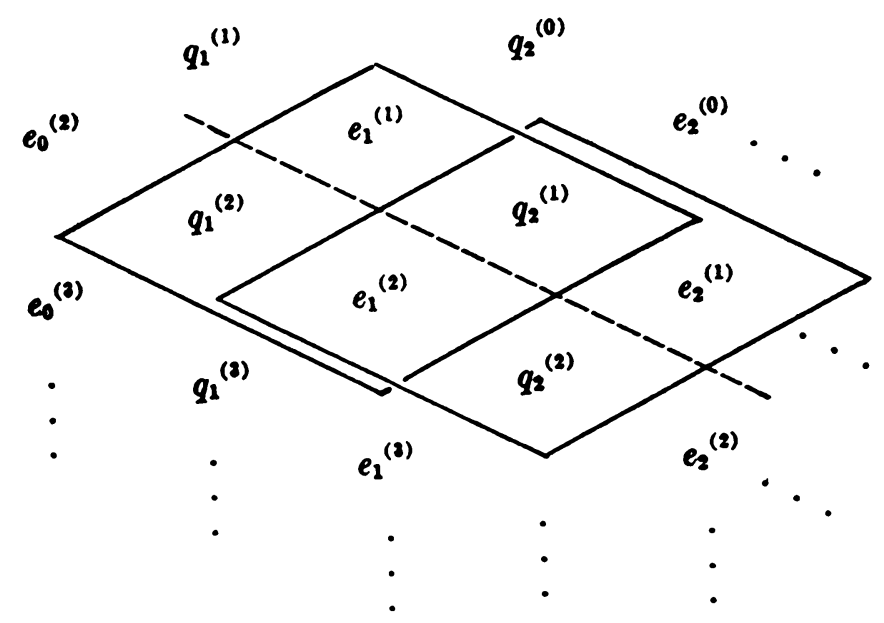

the various quantities occurring in equations (3.3.7) and (3.3.8) are then seen to be located at the corners of a system of lozenges, two of which have been sketched. The computations are started with the initial conditions

$$
e^{(m)}=0, \quad q_{1}^{(m)}=c_{m+1} / c_{m} .
$$

The system of equations (3.3.7) and (3.3.8) is the Quotient-Difference $(q-d)$ algorithm. Formulas relating to it have been given by a number of authors [7], [8], $[3$, p. 382], [9], [10], [11]. Properties of this algorithm which are of critical interest in the theory of numerical analysis have been exploited by Rutishauser in his expository study [10]. An interesting generalization of the algorithm has recently been given by Bauer [12]. Once the quantities $q_{s}{ }^{(m)}, e_{s}^{(m)} s=1,2, \cdots$ have been derived, the system of polynomials $o_{s}{ }^{(m)}(z), p_{s}{ }^{(m)}(z)$ and the functions for a fixed value of $m$, may be constructed by use of equations (3.3.1), (3.1.16), (3.1.17), and (3.1.18). 


\subsection{Further Polynomial Recursion Systems}

Two further results which relate the quantities $q_{s}^{(m)}, e_{0}^{(m)}$ and the polynomials $p_{r}^{(m)}(z)$ are $[10$, p. 11]

$$
p_{r}^{(m+1)}(z)=p_{r}{ }^{(m)}(z)-e_{r}^{(m)} p_{r-1}^{(m+1)}(z)
$$

and

$$
p_{r}^{(m)}(z)=z p_{r-1}^{(m+1)}(z)-q_{r}^{(m)} p_{r-1}^{(m)}(z)
$$

It follows trivially from the definitions (3.1.9) and (3.1.13) that

$$
\begin{aligned}
& z 0_{r}{ }^{(m)}(z)-o_{r}{ }^{(m+1)}(z)-e_{r}{ }^{(m)} o_{r-1}^{(m+1)}(z)=c_{m} p_{r}{ }^{(m)}(z) \\
& o_{r}^{(m)}(z)+q_{r}^{(m)} o_{r-1}^{(m)}(z)-o_{r-1}^{(m+1)}(z)=c_{m} p_{r-1}^{(m+1)}(z) \\
& r_{r}{ }^{(m+1)}(z)=r_{r}{ }^{(m)}(z)-e_{r}{ }^{(m)} r_{r-1}^{(m+1)}(z) \\
& r_{r}^{(m)}(z)=z r_{r-1}^{(m+1)}(z)-q_{r}^{(m)} r_{r-1}^{(m)}(z)
\end{aligned}
$$

and after some manipulation, that

$$
\begin{gathered}
e_{r}^{(m)} z p_{r-1}^{(m+1)}(z)+\left(q_{r+1}^{(m)}-e_{r+1}^{(m-1)}-z\right) p_{r}{ }^{(m)}(z)+p_{r+1}^{(m-1)}(z)=0 \\
q_{r+1}^{(m-1)} p_{r}{ }^{(m-1)}(z)+\left(e_{r}{ }^{(m)}-q_{r+1}^{(m-1)}-z\right) p_{r}{ }^{(m)}(z)+z p_{r}{ }^{(m+1)}(z)=0 \\
e_{r}^{(m)} z r_{r-1}^{(m+1)}(z)+\left(q_{r+1}^{(m)}-e_{r+1}^{(m+1)}-z\right) r_{r}{ }^{(m)}(z)+r_{r+1}^{(m-1)}(z)=0 \\
q_{r+1}^{(m-1)} r_{r}{ }^{(m-1)}(z)+\left(e_{r}{ }^{(m)}-q_{r+1}^{(m-1)}-z\right) r_{r}{ }^{(m)}(z)+z r_{r}{ }^{(m+1)}(z)=0 \\
e_{r}^{(m)} o_{r-1}^{(m+1)}(z)+\left(q_{r+1}^{(m)}-e_{r+1}^{(m-1)}-z\right) o_{r}{ }^{(m)}(z)+z o_{r+1}^{(m-1)}(z) \\
\quad+c_{m} e_{r}^{(m)} p_{r-1}^{(m+1)}(z)-c_{m-1} p_{r+1}^{(m-1)}(z)=0 \\
q_{r}^{(m-1)} z o_{r}{ }^{(m-1)}(z)+\left(e_{r}{ }^{(m)}-q_{r}{ }^{(m-1)} z\right) o_{r}{ }^{(m)}(z)+o_{r}{ }^{(m+1)}(z) \\
\quad+c_{m} p_{r}{ }^{(m)}(z)-c_{m-1} q_{r}{ }^{(m-1)} p_{r}{ }^{(m-1)}(z)=0 .
\end{gathered}
$$

The transcribed recursions (3.1.16) and (3.1.18), namely

$$
\begin{aligned}
& p_{r+1}^{(m)}(z)=\left\{z-q_{r+1}^{(m)}-e_{r}{ }^{(m)}\right\} p_{r}{ }^{(m)}(z)-e_{r}{ }^{(m)} q_{r}{ }^{(m)} p_{r-1}^{(m)}(z) \\
& r_{r+1}^{(m)}(z)=\left\{z-q_{r+1}^{(m)}-e_{r}{ }^{(m)}\right\} r_{r}{ }^{(m)}(z)-e_{r}{ }^{(m)} q_{r}{ }^{(m)} r_{r-1}^{(m)}(z)
\end{aligned}
$$

are recursions involving quantities lying upon diagonals of the $E$-array, (3.4. $)$ and (3.4.9) and (3.4.10) involve quantities lying upon rows and columns of the $E$-array respectively. Both pairs of recursions (3.4.7) and (3.4.9), and (3.4.8) and (3.4.10) could be used to construct continued fraction expansions whose convergents lie upon a row or a column of the $E$-array, and indeed, by suitable use of the recursions (3.4.1), (3.4.2), (3.4.5) and (3.4.6), continued fractions may be constructed whose convergents lie upon arbitrary paths in the $E$-array, but numerical evidence shows that practical interest attaches only to the continued fractions of the form (3.3.2).

\subsection{The Euler-Minding Relations}

Eliminating the quantity $z-\alpha_{r}{ }^{(m)}$ between the recursions 


$$
\begin{aligned}
p_{r+1}^{(m)}(z) & =\left(z-\alpha_{r}{ }^{(m)}\right) p_{r}^{(m)}(z)-\beta_{r-1}^{(m)} p_{r-1}^{(m)}(z) \\
r_{r+1}^{(m)}(z) & =\left(z-\alpha_{r}{ }^{(m)}\right) r_{r}{ }^{(m)}(z)-\beta_{r+1}^{(m)} r_{r-1}^{(m)}(z)
\end{aligned}
$$

there follows

$$
r_{r+1}^{(m)}(z) p_{r}{ }^{(m)}(z)-p_{r+1}^{(m)}(z) r_{r}{ }^{(m)}(z)=\beta_{r-1}^{(m)}\left\{r_{r}{ }^{(m)}(z) p_{r-1}^{(m)}(z)\right.
$$

$$
\left.=c_{m} \beta_{0}^{(m)} \beta_{1}{ }^{(m)} \cdots \beta_{r-1}^{(m)}-r_{r-1}^{(m)}(z) p_{r}{ }^{(m)}(z)\right\}
$$

or, dividing equation (3.5.3) through by $p_{r+1}^{(m)}(z) p_{r}^{(m)}(z)$ writing, $1,2 \cdots n$ for $r+1$, and adding the resulting equations

$$
\frac{r_{n}^{(m)}(z)}{p_{n}^{(m)}(z)}=r_{0}^{(m)}(z)+\sum_{n=1}^{r z} \frac{c_{m} \beta_{0}^{(m)} \cdots \beta_{r-2}^{(m)}}{p_{r-1}^{(m)}(z) p_{r}^{(m)}(z)}
$$

the Euler-Minding relations $[2$, p. 16], [3, p. 17], for the continued fraction (3.1.21). 3.6 A Non-linear Recursion

The rational function $E_{n}{ }^{(m)}(z)$ may also be computed by using a further result in the theory of continued fractions, namely that $n$th convergent

$$
C_{n}=\frac{a_{1}}{b_{1}+} \frac{a_{2}}{b_{2}+} \cdots \frac{a_{n}}{b_{n}}
$$

of the continued fraction

$$
\frac{a_{1}}{b_{1}+} \frac{a_{2}}{b_{2}+} \cdots \frac{a_{n}}{b_{n}+} \cdots
$$

may be computed by evaluating the sequence

$$
D_{1}=\frac{a_{n}}{b_{n}}, \quad D_{r+1}=\frac{a_{n-r}}{b_{n-r}+D_{r}} \quad r=1,2, \cdots, n-1,
$$

when

$$
D_{n}=C_{n} \text {. }
$$

Writing

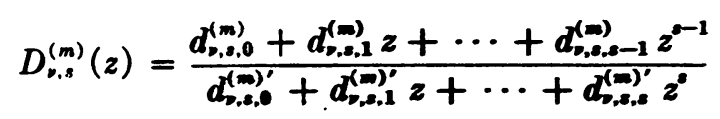

it is seen that the rational function $o_{\nu}{ }^{(m)}(z) / p_{\nu}{ }^{(m)}(z)$ may be constructed by evaluating the sequence

$$
\begin{aligned}
D_{v, 1}^{(m)}(z) & =\frac{\beta_{v-2}^{(m)}}{z-\alpha_{v-1}^{(m)}} \\
D_{v, s+1}^{(m)}(z) & =\frac{\beta_{v-t-2}^{(m)}}{z-\alpha_{v-t-1}^{(m)}-D_{v, s}^{(m)}(z)}
\end{aligned}
$$

when

$$
D_{v, \nu}^{(m)}(z)=0_{\nu}^{(m)}(z) / p_{v}^{(m)}(z) .
$$

The quotient $E_{n}{ }^{(m)}(z)$ may then be constructed by means of equation (3.1.24). 
It will be observed however, that whereas the evaluation of the sequences (3.1.16), (3.1.18) and (3.5.4) are progressive in the sense that at each stage there results the evaluation of a new function $p_{r}{ }^{(m)}(z), r_{r}{ }^{(m)}(z)$ or $E_{r}{ }^{(m)}(z)$, the evaluation of the whole sequence $D_{\nu, s}^{(m)}(z) \quad s=1, \cdots, \nu$ results in the computation of but one quotient $E_{\nu}{ }^{(m)}(z)$.

\subsection{The $e_{m}\left(S_{n}\right)$ Transformation [13] [14]}

If the partial sums $S_{m}$ of the infinite series $\sum_{r=0}^{\infty} c_{r} z^{-r-1}$ are defined by

$$
S_{m}=\sum_{r=0}^{m-1} c_{r} z^{-r-1} \quad r=1,2, \cdots, S_{0}=0
$$

then

$$
\begin{aligned}
\Delta S_{m} & =S_{m+1}-S_{m} \\
& =c_{m} z^{-m-1} .
\end{aligned}
$$

Equation (2.17), by means of suitable operations upon rows and columns of the determinantal expressions which arise from the application of equation (3.1.13) to the expression (3.1.15), may be manipulated into the form

$$
E_{n}^{(m)}(z)=\left|\begin{array}{cccc}
S_{m} & S_{m+1} & \cdots & S_{m+n} \\
\Delta S_{m} & \Delta S_{m+1} & \cdots & \Delta S_{m+n} \\
\Delta S_{m+1} & \Delta S_{m+2} & \cdots & \Delta S_{m+n+1} \\
: & : & \cdots & : \\
\Delta S_{m+n-1} & \Delta S_{m+n} & \cdots & \Delta S_{m+2 n-1} \\
\hline 1 & 1 & \cdots & 1 \\
\Delta S_{m} & \Delta S_{m+1} & \cdots & \Delta S_{m+n} \\
\Delta S_{m+1} & \Delta S_{m+2} & \cdots & \Delta S_{m+n+1} \\
: & : & \cdots & : \\
\Delta S_{m+n-1} & \Delta S_{m+n} & \cdots & \Delta S_{m+2 n-1}
\end{array}\right|
$$

The determinantal quotient (3.7.3) was denoted by Shanks [1t] as $e_{n}\left(S_{m+n}\right)$ and was investigated, primarily as a non-linear sequence to sequence transformation, by him.

\subsection{The E-Algorithm}

The various quantities $E_{n}{ }^{(m)}(z) m=\mathrm{C}, 1, \cdots ; n=0,1, \cdots$, may be constructed by means of a simple recursive algorithm due to Wynn [1j]. According to this, quantities $E_{n+1 / 2}^{(m)}(z) \quad n=-1,0,1, \cdots$ are introduced, and using as boundary values $E_{-1 / 2}^{(m)}(z)=0, E_{0}{ }^{(m)}(z)=S_{m}, \quad m=0,1, \cdots$ further quantities $E_{s}^{(m)}(z)$ $s=\frac{1}{2}, 1, \frac{3}{2}, \cdots ; m=0,1, \cdots$ are constructed by means of the recursion

$$
E_{s}^{(m)}(z)=E_{s-1}^{(m+1)}(z)+\frac{1}{E_{s-1 / 2}^{(m+1)}(z)-E_{s-1 / 2}^{(m)}(z)}
$$

(In the original exposition of this algorithm, [15], the right side of (3.7.3) was regarded as the result of a non-linear transformation of the quantities $S_{r}$, and the notation $E_{n+1 / 2}^{(m)}(z)=\epsilon_{2 n+1}\left(S_{m}\right), E_{n}{ }^{(m)}(z)=\epsilon_{2 n}\left(S_{m}\right)$, was used.)

Since all the quantities $E_{s}{ }^{(m)}(z)$ are rational functions of $z$, and equation (3.8.1) may be manipulated into such a form as would require only the multiplication. 
addition, and subtraction of polynomial expressions, the $\epsilon$-algorithm provides a method for computing the constituents of the $E$-array.

A certain measure of economy in the use of the $\epsilon$-algorithm for this purpose may however be effected by writing down explicit formulas for some of the quantities occurring in equation (3.8.1). Accordingly, as may easily be verified

$$
\begin{aligned}
& E_{n+1}^{(m)}(z)-E_{n}^{(m+1)}(z)=\frac{1}{E_{n+1 / 2}^{(m+1)}(z)-E_{n+1 / 2}^{(m)}(z)} \\
& =\frac{\left|\begin{array}{cccc}
c_{m} & c_{m+1} & \cdots & c_{m+n} \\
c_{m+1} & c_{m+2} & \cdots & c_{m+n+1} \\
: & \vdots & & \vdots \\
c_{m+n} & c_{m+n+1} & \cdots & c_{m+2 n}
\end{array}\right|\left|\begin{array}{cccc}
c_{m+1} & c_{m+2} & \cdots & c_{m+n+1} \\
c_{m+2} & c_{m+3} & \cdots & c_{m+n+2} \\
: & \vdots & & \vdots \\
c_{m+n+1} & c_{m+n+2} & \cdots & c_{m+2 n+1}
\end{array}\right|}{\left|\begin{array}{cccc}
1 & z & \cdots & z^{n+1} \\
c_{m} & c_{m+1} & \cdots & c_{m+n+1} \\
\vdots & \vdots & & \vdots \\
c_{m+n} & c_{m+n+1} & \cdots & c_{m+2 n+1}
\end{array}\right|\left|\begin{array}{cccc}
1 & z & \cdots & z^{n} \\
c_{m+1} & c_{m+2} & \cdots & c_{m+n+1} \\
: & \vdots & & \vdots \\
c_{m+n} & c_{m+n+1} & \cdots & c_{m+2 n}
\end{array}\right|} \\
& =\frac{c_{m+1} \prod_{\mu=1}^{n} e_{\mu}^{(m+1)} q_{\mu}^{(m+1)}}{p_{n+1}^{(m)}(z) p_{n}^{(m+1)}(z)} \\
& =\frac{c_{m+1} \beta_{0}^{(m+1)} \beta_{1}^{(m+1)} \cdots \beta_{n-1}^{(m+1)}}{p_{n+1}^{(m)}(z) p_{n}^{(m+1)}(z)}
\end{aligned}
$$

$$
E_{n+1 / 2}^{(m)}(z)-E_{n-1 / 2}^{(m+1)}(z)=\frac{1}{E_{n}^{(m+1)}(z)-E_{n}^{(m)}(z)}
$$

$$
\begin{aligned}
& =\frac{\left|\begin{array}{cccc}
1 & z & \cdots & z^{n} \\
c_{m} & c_{m+1} & \cdots & c_{m+n} \\
: & : & & : \\
c_{m+n-1} & c_{m+n} & \cdots & c_{m+2 n-1}
\end{array}\right|\left|\begin{array}{cccc}
1 & z & \cdots & z^{n} \\
c_{m+1} & c_{m+2} & \cdots & c_{m+n+1} \\
: & : & & \vdots \\
c_{m+n} & c_{m+n+1} & \cdots & c_{m+2 n}
\end{array}\right|}{\left|\begin{array}{cccc}
c_{m} & c_{m+1} & \cdots & c_{m+n} \\
c_{m+1} & c_{m+2} & \cdots & c_{m+n+1} \\
: & : & & \vdots \\
c_{m+n} & c_{m+n+1} & \cdots & c_{m+2 n}
\end{array}\right|\left|\begin{array}{cccc}
c_{m+1} & c_{m+2} & \cdots & c_{m+n} \\
c_{m+2} & c_{m+3} & \cdots & c_{m+n+1} \\
c_{m+n} & c_{m+n+1} & \cdots & c_{m+2 n-1}
\end{array}\right|} \\
& =\frac{p_{n}^{(m)}(z) p_{n}^{(m+1)}(z)}{c_{m} \prod_{\mu=0}^{n} e_{\mu}^{(m)} q_{\mu}^{(m)}}=\frac{p_{n}^{(m)}(z) p_{n}^{(m+1)}(z)}{c_{m} \beta_{0}^{(m)} \beta_{1}^{(m)} \cdots \beta_{n-1}^{(m)}} \\
& E_{n+1 / 2}^{(m)}(z)=-\frac{\left|\begin{array}{ccccc}
0 & 1 & z & \cdots & z^{n} \\
1 & c_{m} & c_{m+1} & \cdots & c_{m+n} \\
z & c_{m+1} & c_{m+2} & \cdots & c_{m+n+1} \\
\vdots & : & \vdots & & \vdots \\
z^{n} & c_{m+n} & c_{m+n+1} & \cdots & c_{m+2 n}
\end{array}\right|}{\left|\begin{array}{cccc}
c_{m} & c_{m+1} & \cdots & c_{m+n} \\
c_{m+1} & c_{m+2} & \cdots & c_{m+n+1} \\
\vdots & \vdots & & \vdots \\
c_{m+n} & c_{m+n+1} & \cdots & c_{m+2 n}
\end{array}\right|} z^{m+1}
\end{aligned}
$$


It is immediately seen that the quantities $E_{n+1 / 2}^{(m)}(z)$ are, apart from a factor of $z^{m+1}$, polynomials of the $2 n$th degree in $z$, and may be written

$$
E_{n+1 / 2}^{(m)}(z)=z^{m+1} \sum_{j=0}^{-2 n} \epsilon_{n, 8}^{(m)} z^{*}
$$

In this instance a large part of the computational effort which, upon the assumption that $E_{s}{ }^{(m)}(z) \quad s=\frac{1}{2}, 1, \cdots$ is a general rational function of $z$, would go into the computation of coefficients which are known to be zero, may be avoided.

\subsection{Example: The Incomplete Gamma Function of Large Argument}

Certain aspects of the preceding theory are illustrated by the integral

$$
\begin{aligned}
\{\Gamma(\alpha)\}^{-1} \int_{0}^{\infty} \frac{t^{\alpha-1} e^{-t}}{z-t} d t & \sim \frac{1}{z}+\frac{\alpha}{z^{2}}+\frac{\alpha(\alpha+1)}{z^{3}}+\cdots \\
& =\frac{1}{z-\alpha-\alpha} \frac{1 \cdot \alpha}{2+\alpha-z-} \cdots \frac{r(\alpha+r-1)}{2 r+\alpha-z-} \cdots
\end{aligned}
$$

Here

$$
c_{m}=\frac{\Gamma(\alpha+m)}{\Gamma(\alpha)}
$$

and as is easily verified, the quantities $q_{r}^{(m)}, e_{r}^{(m)}$ of the $q-d$ algorithm, are given in this ease by

$$
q_{r}^{(m)}=\alpha+m+r-1, \quad e_{r}^{(m)}=r
$$

further in the notation of equation (3.1.6)

$$
p_{r}^{(0)}(z)=L_{r}^{(\alpha-1)}(z)
$$

where $L_{r}^{(\alpha)}(z)$, the Laguerre polynomial of order $\alpha$ and degree $n$ satisfies the recursion

$$
(n+1) L_{n+1}^{(\alpha)}(z)-(2 n+1+\alpha-z) L_{n}^{(\alpha)}(z)+(n+\alpha) L_{n-1}^{(\alpha)}(z)=0 .
$$

Replacing $z$ by $-z,(3.9 .1)$ gives the expansion, suitable for large values of the argument, for the incomplete gamma function

$$
\begin{aligned}
\Gamma(1-\alpha, z) \sim \frac{1}{z}-\frac{\alpha}{z^{2}} & +\frac{\alpha(\alpha+1)}{z^{3}}-\cdots \\
& =\frac{1}{z+\alpha-} \frac{1 \cdot \alpha}{z+\alpha+2-} \cdots \frac{r(\alpha+r-1)}{z+\alpha+2 r-} \cdots
\end{aligned}
$$

The initial members of the $E$-array for this function are given in Table 1 .

When $\alpha=1$, (3.9.6) becomes

$$
\begin{aligned}
e^{z} \int_{z}^{\infty} \frac{e^{-t}}{t} d t \sim-\sum_{r=0}^{\infty} r !(-z)^{-r-1} & \\
& =\frac{1}{z+1-\frac{1^{2}}{z+3-}} \cdots \frac{r^{2}}{z+2 r+1-\cdots}
\end{aligned}
$$

Numerical values of the entries in Table I when $\alpha=z=1$ are displayed in Table 2. 


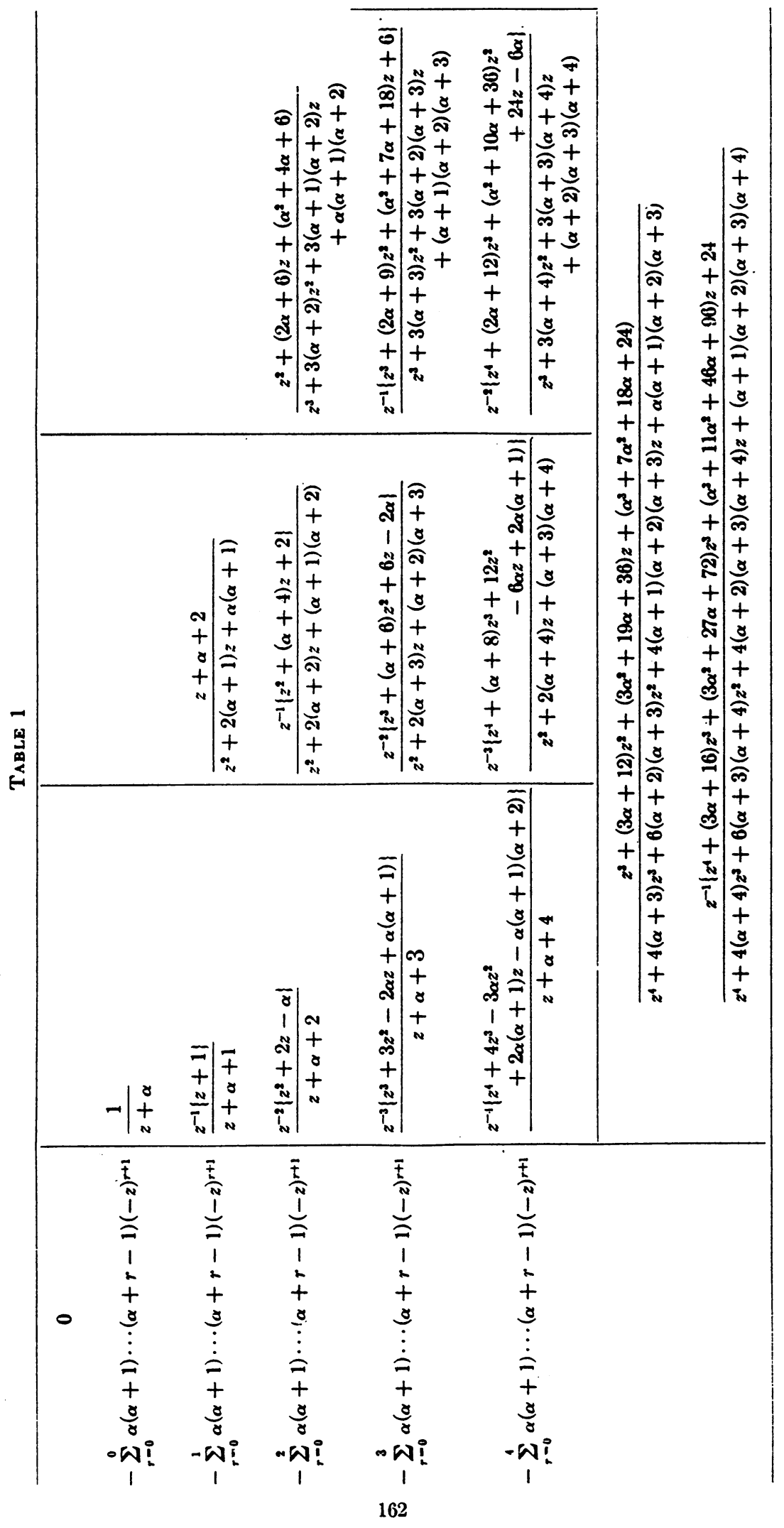


TABLE 2

\begin{tabular}{rlllllll}
\hline 0 & & & & & & \\
1 & 0.5 & & & $e^{1} \int_{1}^{\infty} \frac{e^{-t}}{t} d t=0.59634786$ \\
0 & 0.6 & 0.5714 & 2857 & & & & \\
+2 & 0.5 & $0 . \dot{6153}$ & 8462 & 0.5882 & 3529 & & \\
-4 & 0.8 & 0.5714 & $28 \dot{5} 7$ & 0.6027 & 3973 & 0.5933 & 0144 \\
+20 & 0.0 & 0.7096 & 7742 & 0.5882 & 3529 & 0.5988 & 0240 \\
\hline
\end{tabular}

Some further particular cases of (3.9.6) are the Complementary Error Function

$$
e^{z^{2}} \int_{z}^{\infty} e^{-t^{2}} d t=z\left\{\frac{1}{2 z^{2}+1-2 z^{2}+5-} \cdots \frac{r(r+1)}{2 z^{2}+4 r+1-\cdots}\right\}
$$

and Dawson's Integral

$$
\begin{aligned}
& e^{-z^{2}} \int_{z}^{\infty} e^{t^{2}} d t \\
& \quad \sim-\frac{e^{-z^{2}} \pi^{1 / 2} i}{2}+z\left\{\frac{1}{\left.2 z^{2}-1-\frac{1 \cdot 2}{2 z^{2}-5-} \cdots \frac{r(r+1)}{2 z^{2}-4 r-1-} \cdots\right\} .}\right.
\end{aligned}
$$

(This continued fraction is of course divergent, since its convergents are all real and the integral is real. However there remains an uncancelled imaginary constituent upon the right hand side of (3.9.9). Nevertheless the continued fraction (3.9.9) exhibits a sort of semi-convergent behavior. It is to be preferred for the computation of Dawson's integral for large values of the argument to the expansion (4.1.12) which is convergent. Indeed the expansion

$$
\frac{1}{2 z-\frac{2}{2 z-}} \frac{4}{2 z-} \frac{6}{2 z-} \cdots
$$

the even part of which is the expansion (3.9.9), was used by Barkley Rosser for the tabulation of the Error Function for complex argument [16].) The Sine and Cosine Integrals are given by

$$
C i(z)+i \operatorname{si}(z)=-(\cos z-i \sin z)
$$

The Fresnel Integrals

$$
C(z)=(2 \pi)^{-1 / 2} \int_{0}^{z} t^{-1 / 2} \cos t d t, \quad S(z)=(2 \pi)^{-1 / 2} \int_{0}^{z} t^{-1 / 2} \sin t d t
$$

may be computed by writing

$$
C(z)=\frac{1}{2}-(2 \pi)^{-1 / 2} C\left(z, \frac{1}{2}\right) \quad S(z)=\frac{1}{2}-(2 \pi)^{-1 / 2} S\left(z, \frac{1}{2}\right)
$$

where

$$
\begin{aligned}
C\left(z, \frac{1}{2}\right)- & i S\left(z, \frac{1}{2}\right) \\
& =(\sin z-i \cos z) z^{1 / 2} \frac{2}{2 i z+1-} \frac{1 \cdot 2}{2 i z+5-} \cdots \frac{r(r+1)}{2 i z+4 r+1-\cdots\} .} .
\end{aligned}
$$


4. The Pade Table. The remarks contained in $\$ 2$ indicate that the upper half of the Pade Table may be constructed by methods described for the construction of the $E$-array.

It would appear, since the system of orthogonal polynomials $p_{r}^{(m)}(z)$ does not exist for negative values of $m$, that the use of the results in the theory of orthogonal polynomials as described, for the purpose of constructing the lower half of the Padé table, is not possible. Nevertheless, if the first three polynomials can be determined in some other way, the sequence may be continued by use of the results as described, since the unknown quantities $\beta_{0}^{(m)}, \beta_{1}^{(m)}, \cdots, \beta_{-m-1}^{(m)}, \alpha_{0}^{(m)}, \alpha_{1}^{(m)}, \cdots$, $\alpha_{-m}^{(m)}$ where $m$ is negative, disappear from the resulting computations.

In terms of $q-d$ scheme, as defined by equations (3.3.7), (3.3.8), the rational expressions

$$
\frac{U_{0, m-1}(x)}{V_{0, m-1}(x)}, \quad \frac{U_{0, m}(x)}{V_{0, m}(x)}, \quad \frac{U_{1, m}(x)}{V_{1, m}(x)}, \quad \frac{U_{1, m+1}(x)}{V_{1, m+1}(x)}, \ldots
$$

are the successive convergents of the continued fraction

$$
\sum_{i=0}^{m-1} c_{n} x^{*}+\frac{c_{m} x^{m}}{1-} \frac{q_{1}^{(m)} x}{1-} \frac{e_{1}^{(m)} x}{1-} \frac{q_{2}^{(m)} x}{1-} \frac{e_{2}^{(m)} x}{1-} \cdots
$$

while the rational expressions

$$
\frac{U_{0, m-1}(x)}{V_{0, m-1}(x)}, \quad \frac{U_{1, m}(x)}{V_{1, m}(x)}, \quad \frac{U_{2, m+1}(x)}{V_{2, m+1}(x)}, \cdots
$$

are the successive convergents of the even part of expansion (4.1), namely,

$$
\begin{aligned}
& \sum_{s=0}^{m-1} c_{s} x^{s}+\frac{c_{m} x^{m}}{1-q_{1}^{(m)} x-}-\frac{e_{1}^{(m)} q_{1}^{(m)} x^{2}}{1-\left(q_{2}^{(m)}+e_{1}^{(m)}\right) x-} \\
& \cdots \frac{e_{r}^{(m)} q_{r}^{(m)} x^{2}}{1-\left(q_{r+1}^{(m)}+e_{r}^{(m)}\right) x-} \cdots
\end{aligned}
$$

If the series $\sum_{s=0}^{\infty} d_{x} t^{*}$ is derived by means of the relation

$$
\left(\sum_{s=0}^{\infty} c_{s} x^{*}\right)\left(\sum_{s=0}^{\infty} d_{s} \cdot x^{s}\right)=1
$$

and the quantities derived by applying the relationships of the $q-d$ algorithm to the quantities $d_{*}, s=0,1, \cdots$, are denoted by $q_{r}^{(m) \prime}, e_{r}^{(m) \prime}$, then the rational functions

$$
\frac{U_{m-1,0}(x)}{V_{m-1,0}(x)}, \quad \frac{U_{m .0}(x)}{V_{m .0}(x)}, \quad \frac{U_{m, 1}(x)}{V_{m, 1}(x)}, \quad \frac{U_{m+1,1}(x)}{V_{m+1,1}(x)}, \cdots
$$

are the successive convergents of the continued fraction

$$
\frac{1}{d_{0}+d_{1} x+d_{2} x^{2}+\cdots+d_{m-1} x^{m-1}}+\frac{d_{m} x^{m}}{1-} \frac{q_{1}^{(m) \prime} x}{1-} \frac{e_{1}^{(m) \prime} x}{1-} \frac{q_{2}^{(m) \prime} x}{1-} \frac{e_{2}^{(m) \prime} x}{1-} \cdots
$$

If the quantities $d_{s}, s=0,1, \cdots$, defined by equation (4.3) are required in isolation, they are most efficiently derived by solving the scheme

$$
\sum_{s=0}^{s=r} d_{s} c_{r-s}= \begin{cases}1 & r=0 \\ 0 & r \neq 0\end{cases}
$$


If, however, the quantities ${q_{r}}^{(m)^{\prime}}, e_{r}{ }^{(m)^{\prime}}$ are also required, some economy in the computation may be achieved by use of a result in the theory of continued fractions [16], [2, p. 332], namely that if

$$
\beta(x)=\frac{c_{0}}{1-} \frac{q_{1}^{(0)} x}{1-} \frac{e_{1}^{(0)} x}{1-} \frac{q_{2}^{(0)} x}{1-} \cdots
$$

and

$$
\{\beta(x)\}^{-1}=\frac{d_{0}}{1-} \frac{q_{1}^{(0)^{\prime}} x}{1-} \frac{e_{1}^{(0)^{\prime}} x}{1-} \frac{q_{2}^{(0)^{\prime}} x}{1-} \frac{\left.e_{2}^{(0)}\right)^{\prime} x}{1-} \cdots,
$$

then

$$
\begin{aligned}
& d_{0}=c_{0}{ }^{-1}, \quad q_{1}{ }^{(0)^{\prime}}=-q_{1}{ }^{(0)}, \quad e_{1}{ }^{(0)^{\prime}}=q_{1}{ }^{(0)}+e_{1}{ }^{(0)}, \\
& e_{r}{ }^{(0)} q_{r+1}^{(0)^{\prime}}=e_{r}{ }^{(0)} q_{r+1}^{(0)}, \quad e_{r+1}^{(0)^{\prime}}+q_{r+1}^{(0)^{\prime}}=e_{r+1}^{(0)}+q_{r+1}^{(0)},
\end{aligned}
$$

In the determination of the quantities $q_{r}{ }^{(m)}, e_{r}{ }^{(m)}$ the $q-d$ scheme is built up from left to right by means of the $q-d$ relationships (3.3.7) and (3.3.8), while in the determination of the quantities $q_{r}^{(m)^{\prime}}, e_{r}{ }^{(m)^{\prime}}$, the diagonal $q_{1}{ }^{(0)^{\prime}}, e^{(0)^{\prime}}, q_{2}{ }^{(0)^{\prime}}, e_{2}{ }^{(0)^{\prime}}, \ldots$ is determined by equations (4.7), and the $q-d$ relationships are used to build up the $q-d$ scheme from right to left.

It is to be expected that the recursion formulas between the systems of functions $r_{n}{ }^{(m)}(z), p_{n}{ }^{(m)}(z)$ are complemented by recursions between the polynomials $U_{\mu, \nu}(x), V_{\mu, \nu}(x)$ occurring in the Padé table. Indeed Frobenius [17] has given such a system of recursion formulas. Adopting his notation, write

$$
\begin{aligned}
& c_{\mu, \nu}=\left|\begin{array}{cccc}
c_{\mu-\nu+1} & c_{\mu-\nu+2} & \cdots & c_{\mu} \\
c_{\mu-v+2} & c_{\mu-v+3} & \cdots & c_{\mu+1} \\
: & \vdots & & \vdots \\
c_{\mu} & c_{\mu+1} & \cdots & c_{\mu+\nu-1}
\end{array}\right| \\
& T_{\mu, \nu}=\left|\begin{array}{ccccc}
c_{\mu-\nu+1} & c_{\mu-\nu+2} & \cdots & c_{\mu} & c_{\mu-\nu} x^{\mu}+c_{\mu-\nu-1} x^{\mu-1}+\cdots \\
c_{\mu-\nu+2} & c_{\mu-\nu+3} & \cdots & c_{\mu+1} & c_{\mu-\nu+1} x^{\mu}+c_{\mu-\nu} x^{\mu-1}+\cdots \\
: & : & & : & : \\
c_{\mu+1} & c_{\mu+2} & \cdots & c_{\mu+\nu} & c_{\mu} x^{\mu}+c_{\mu-1} x^{\mu-1}+\cdots
\end{array}\right| \\
& S_{\mu, \nu}=\left|\begin{array}{ccccc}
c_{\mu-\nu+1} & c_{\mu-\nu+2} & \cdots & c_{\mu} & x^{\nu} \\
c_{\mu-\nu+2} & c_{\mu-\nu+3} & \cdots & c_{\mu+1} & x^{\nu-1} \\
: & : & & : & : \\
c_{\mu+1} & c_{\mu+2} & \cdots & c_{\mu+\nu} & 1
\end{array}\right|
\end{aligned}
$$

so that

$$
\begin{aligned}
c_{\mu, \nu} & =H_{\mu-\nu+1}^{(\nu)} \\
\beta(x) S_{\mu, \nu}-T_{\mu, \nu} & =c_{\mu+1, \nu+1} x^{\mu+\nu+1}+\cdots \\
T_{\mu, \nu} & =U_{\mu, \nu}(x) c_{\mu+1, \nu} \\
S_{\mu, \nu} & =V_{\mu, \nu}(x) c_{\mu+1, \nu}
\end{aligned}
$$

He then gives the following system of homogeneous recursion formulas, where $R_{\mu, \nu}$ is taken to mean either $S_{\mu, \nu}$ or $T_{\mu, \nu}$ 


$$
\begin{gathered}
c_{\mu+1, \nu} R_{\mu-1, \nu}-c_{\mu, \nu+1} R_{\mu, \nu-1}=c_{\mu, \nu} R_{\mu, \nu} \\
c_{\mu, \nu+1} R_{\mu+1, \nu}-c_{\mu+1, \nu} R_{\mu, \nu+1}=c_{\mu+1, \nu+1} x R_{\mu, \nu} \\
c_{\mu+1, \nu} R_{\mu, \nu}-c_{\mu, \nu} R_{\mu+1, \nu}=c_{\mu+1, \nu+1} x R_{\mu, \nu-1} \\
c_{\mu, \nu+1} R_{\mu, \nu}-c_{\mu, \nu} R_{\mu, \nu+1}=c_{\mu+1, \nu+1} x R_{\mu-1, \nu} \\
c_{\mu, \nu} c_{\mu, \nu+1} R_{\mu+1, \nu}-\left(c_{\mu, \nu+1} c_{\mu+1, \nu}+c_{\mu, \nu} c_{\mu+1, \nu+1} x\right) \\
\cdot R_{\mu, \nu}+c_{\mu+1, \nu} c_{\mu+1, \nu+1} x R_{\mu-1, \nu}=0 \\
c_{\mu, \nu} c_{\mu+1, \nu} R_{\mu, \nu+1}-\left(c_{\mu, \nu+1} c_{\mu+1, \nu}-c_{\mu, \nu} c_{\mu+1, \nu+1} x\right) \\
\cdot R_{\mu, \nu}+c_{\mu, \nu+1} c_{\mu+1, \nu+1} x R_{\mu, \nu-1}=0 \\
c_{\mu, \nu}^{2} R_{\mu+1, \nu+1}-\left\{c_{\mu, \nu} c_{\mu+1, \nu+1}+\left(c_{\mu+1, \nu+2} c_{\mu, \nu-1}-c_{\mu+2, \nu+2} c_{\mu-1, \nu}\right) x\right\} \\
\cdot R_{\mu, \nu}+c_{\mu+1, \nu+1}^{2} x^{2} R_{\mu-1, \nu-1}=0 .
\end{gathered}
$$

Equation (4.19) is a three term linear homogeneous recursion between the numerators and denominators of constituents of the Pade table lying in the same row, equation (4.20) another such relating to a column. Equation (4.21) relates numerators and denominators of constituents in the Padé table lying along a diagonal.

It will immediately be realized that the triplets of equations (3.4.1), (3.4.5) and (4.17); (3.4.2), (3.4.6) and (4.18); (3.4.8), (3.4.10) and (4.19); (3.4.7), (3.4.9) and $(4.20) ;(3.1 .16),(3.1 .18)$, and $(4.21)$ are identical. Frobenius also gives a further system of inhomogeneous recursions, which do not seem to have immediate practical application. The function

$$
\frac{U_{0, m-1}(x)}{V_{0, m-1}(x)}, \quad \frac{U_{0, m}(x)}{V_{0, m}(x)}, \quad \frac{U_{1, m}(x)}{V_{1, m}(x)}, \cdots
$$

may also be formed by applying the Euler-Minding relations to the continued fraction

$$
\sum_{s=0}^{m-1} c_{s} x^{s}+\frac{c_{m} x^{m}}{1-} \frac{q_{1}^{(m)} x}{1-} \frac{e_{1}^{(m)} x}{1-} \cdots \frac{q_{r}^{(m)} x}{1-} \frac{e_{r}^{(m)} x}{1-} \cdots
$$

in direct analogy to the process preceding equation (3.j.4): further, the functions

$$
\frac{\zeta_{r, m-1+r}(x)}{V_{r, m-1+r}(x)}=\sum_{s=0}^{m-1} c_{s} x^{*}+\frac{c_{m} x^{m}}{1-} \frac{q_{1}^{(m)} x}{1-} \frac{e_{1}^{(m)} x}{1-} \cdots \frac{q_{r}^{(m)} x}{1}
$$

and

$$
\frac{U_{r, m+r}(x)}{V_{r, m+r}(x)}=\sum_{s=0}^{m-1} c_{s} x^{s}+\frac{c_{m} x^{m}}{1-} \frac{q_{1}^{(m)} x}{1-} \frac{e_{1}^{(m)} x}{1-} \cdots \frac{q_{r}^{(m)} x}{1-} \frac{e_{r}^{(m)} x}{1}
$$

may be built up from the non-linear recursions

$$
\begin{aligned}
& C_{n}=b_{0}+\frac{a_{1}}{b_{1}+} \frac{a_{2}}{b_{2}+} \cdots \frac{a_{n}}{b_{n}} \\
& D_{0}=b_{n}, \quad D_{r}=b_{n-r}+\frac{a_{n-r+1}}{D_{r-1}}, \quad D_{n}=C_{n} .
\end{aligned}
$$

The same remarks apply, of course, to the continued fraction 


$$
\sum_{s=0}^{m-1} d_{s} x^{0}+\frac{d_{m} x^{m}}{1-} \frac{q_{1}^{(m) \prime} x}{1-} \frac{e_{1}^{(m) t} x}{1-} \cdots \frac{q_{r}^{(m) t} x}{1-} \frac{e_{r}^{(m) t} x}{1-} \cdots
$$

the functions computed from (4.27) being the sequence

$$
\frac{V_{m-1,0}(x)}{U_{m-1,0}(x)}, \quad \frac{V_{m, 0}(x)}{U_{m, 0}(x)}, \quad \frac{V_{m, 1}(x)}{U_{m, 1}(x)}, \cdots .
$$

The $\epsilon$-algorithm may also be used to construct the Padé table: the partial sums $\sum_{0 \rightarrow 0}^{r} c_{0} x^{0} \quad r=0,1, \cdots$ are placed in the leading column, the quantities $\left(c_{r} x^{x}\right)^{-1}$ $r=1,2, \cdots$ in the second, and the $\epsilon$-algorithm relationships (3.8.1) applied. The functions

$$
\frac{U_{0,8}(x)}{V_{0.8}(x)}, \quad \frac{U_{1,8}(x)}{V_{1,8}(x)}, \quad \frac{U_{2,8}(x)}{V_{2.8}(x)}, \cdots
$$

then appear in the first, third, fifth and further columns respectively; the array must thus be transposed about the leading diagonal if the notation employed in the description of the Pade table is correctly to be observed. The remainder of the Pade table may be computed by applying the same process to the series $\sum_{8=0}^{\infty} d_{s} x^{*}$ it being necessary subsequently to invert the entries in the first, third, fifth and further columns which remain however in their correct position. The entries in the principal diagonal will in this way have been computed twice, the two sets of results should of course agree.

It is not necessary to compute the partial sums of the inverse series $\sum_{s=0}^{\infty} d_{8} x^{3}$, defined by (2.12), in order to construct the whole Padé table by means of the $\epsilon$-algorithm. In the notation of the $\epsilon$-array one adds the boundary values $\epsilon_{2}^{(-,-1)}=$ 0, and this enables both halves of the Pade table to be constructed. (See P. Wr.v., "L'e-algoritmo e la tavola Padé," to appear.)

\subsection{Example: The Incomplete Beta Function}

An example which may be used to illustrate the theory relating to the Padé table is provided by the series

$$
{ }_{2} F_{1}(1, a ; c ; x)=1+\frac{a}{c} x+\frac{a(a+1) x^{2}}{c(c+1)}+\cdots .
$$

The initial members of the Pade table for this function are given in Table 3.

TABLE 3

\begin{tabular}{l|l|l}
\hline 1 & $1+\frac{a}{c} x$ & $1+\frac{a}{c} x+\frac{a(a+1)}{c(c+1)} x^{2}$ \\
$\frac{1}{1-\frac{a}{c} x}$ & $\frac{1+\frac{1(a-c)}{c(c+1)} x}{1-\frac{(a+1)}{(c+1)} x}$ & $\begin{array}{l}1+\frac{2(a-c)}{c(c+2)} x+\frac{1 a(a-c)}{c(c+1)(c+2)} x^{2} \\
1-\frac{(a+2)}{(c+2)} x\end{array}$ \\
$\frac{1}{1-\frac{a}{c} x+\frac{a(a-c)}{c^{2}(c+1)} x^{2}}$ & $\frac{1+\left\{\frac{(a-c)(c+2)-(a+1) c^{2}}{c(c+1)(c+2)}\right\} x}{1-\frac{2(a+1)}{(c+2)} x+\frac{a(a+1)}{(c+1)(c+2)} x^{2}}$ & $\frac{1+\left\{\frac{a}{c}-\frac{2(a+2)}{(c+3)}\right\} x}{1-\frac{2(a+2)}{(c+3)} x+\frac{(a+1)(a+2)(c+3)}{(c+2)(c+3)} x^{2}}$ \\
\hline
\end{tabular}


As is easily verified, the $q-d$ tableau relating to the series (4.1.1) is given in Table 4.

TABLE 4

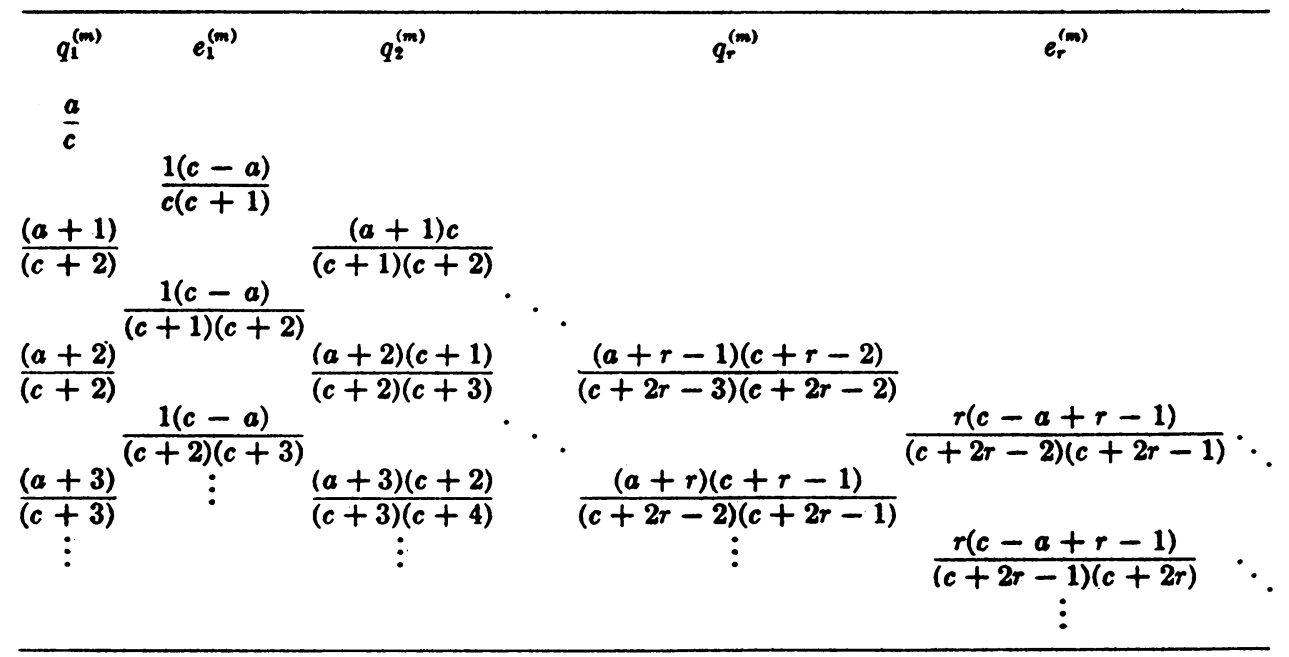

More generally

$$
\begin{aligned}
& q_{r}^{(m)}=\frac{(a+r+m-1)(c+r+m-2)}{(c+2 r+m-3)(c+2 r+m-2)}, \\
& e_{r}^{(m)}=\frac{r(c-a+r-1)}{(c+2 r+m-2)(c+2 r+m-1)}
\end{aligned}
$$

from which follows the continued fraction expansion

$$
\begin{gathered}
1+\sum_{r=1}^{\infty} \frac{a(a+1) \cdots(a+r-1)}{c(c+1) \cdots(c+r-1)} x^{r}=1+\sum_{r=1}^{m} \frac{a(a+1) \cdots(a+r-1)}{c(c+1) \cdots(c+r-1)} x^{r} \\
+\frac{a(a+1) \cdots(a+m)}{c(c+1) \cdots(c+m)} x^{m+1}\left\{\frac{1}{1-\frac{(a+m) x}{c+m-} \frac{1(c-a) x}{c+m+1-}}\right. \\
\left.\cdots \frac{(a+m+r)(c+m+r-1) x}{c+m+2 r-} \frac{(r+1)(c-a+r) x}{c+m+2 r+1-} \cdots\right\}
\end{gathered}
$$

The continued fraction

$$
\begin{aligned}
\frac{1}{1-} \frac{a x}{c-} \frac{1(c-a) x}{c+1-} & \frac{(a+1) c x}{c+2-\frac{2(c-a+1) x}{c+3-}} \\
& \ldots \frac{(a+r)(c+r-1) x}{c+2 r+2-} \frac{(r+1)(c-a+r) x}{c+2 r+2-} \ldots
\end{aligned}
$$

which corresponds to (4.1.3) with $m=0$, was used by Müller [18] in the preparation of a short table of the Incomplete Beta Function. When $a=1, c=2$, and $x$ is replaced by $-x,(4.1 .4)$ reduces to the well known expansion

$$
\log (1+x)=\frac{x}{1+} \frac{1^{2} x}{2+} \frac{1^{2} x}{3+} \frac{2^{2} x}{4+} \cdots \frac{r^{2} x}{2 r+} \frac{r^{2} x}{2 r+1+} \cdots .
$$


Table 3 becomes in this caie (numerical values for $x=1$ are appended) as shown in Table 5 .

TABLE 5

\begin{tabular}{|c|c|c|c|c|c|}
\hline 1 & $1-\frac{1}{2} x$ & $1-\frac{1}{2} x+\frac{1}{3} x^{2}$ & 1.0 & 0.5 & 0.833333 \\
\hline$\frac{1}{1+\frac{1}{2} x}$ & $\frac{1+\frac{1}{6} x}{1+\frac{2}{3} x}$ & $\frac{1+\left\{x-3 x^{2}\right.}{1+\frac{3}{3} x}$ & 0.6 & 0.7 & 0.690476 \\
\hline$\frac{1}{1+\frac{1}{2} x+\frac{1}{12} x^{2}}$ & $\frac{1+\frac{1}{2} x}{1+x+\frac{1}{6} x^{2}}$ & $\frac{1+\mathrm{r}^{7} 0 x+\frac{1}{30} x^{2}}{1+\frac{6}{5} x+{ }_{10}^{3} x^{2}}$ & 0.705882 & 0.692308 & 0.693333 \\
\hline & & & \multicolumn{3}{|c|}{$\log _{e} 2=0.693147 \ldots$} \\
\hline
\end{tabular}

When $a=\frac{1}{2}, c=\frac{3}{2}$ and $x$ is replaced by $-x^{2}$, there follows

(4.1.6) $\quad \arctan x=\frac{x}{1+} \frac{1^{2} x^{2}}{3+} \frac{4 \cdot 1^{2} x^{2}}{5+} \frac{3^{2} x^{2}}{7+} \frac{4 \cdot 2^{2} x^{2}}{9+} \cdots \frac{(2 r+1)^{2} x^{2}}{4 r+3+} \frac{4(r+1)^{2} x^{2}}{4 r+5+} \cdots$

Replacing $x$ by $x / a$ in (4.1.4) and letting $a$ tend to infinity, there follows the continued fraction expansion.

$$
\frac{1}{1-} \frac{x}{c+} \frac{1 x}{c+1-} \frac{c x}{c+2}+\frac{2 x}{c+3}=\cdots \frac{r x}{c+2 r-1-} \frac{(c+r-1) x}{c+2 r+} \cdots
$$

of the incomplete gamma function

$$
c x^{-c} e^{x} \int_{0}^{x} e^{-t} t^{c-1} d t
$$

suitable for small values of $x$. The initial members of the Pade table for this series are shown in Table 6.

\begin{tabular}{l|l|l}
\multicolumn{2}{c}{ TABLE 6 } \\
\hline 1 & 1 & $1+\frac{x}{c}+\frac{x^{2}}{c(c+1)}$ \\
$\frac{1}{1-\frac{x}{c}}$ & $\frac{1+\frac{x}{(c+1)}}{1-\frac{x}{(c+1)}}$ & $\frac{2}{c(c+2)} x+\frac{x^{2}}{c(c+1)(c+2)}$ \\
$\frac{1-\frac{x}{(c+2)}}{1-\frac{x}{c}+\frac{x^{2}}{c^{2}(c+1)}}$ & $\frac{1-\frac{(c-2)}{c(c+2)} x}{1-\frac{2}{(c+2)} x+\frac{x^{2}}{(c+1)(c+2)}}$ & $\frac{1-\frac{(c-3)}{c(c+3)} x+\frac{2}{c(c+1)(c+2)(c+3)} x^{2}}{1-\frac{2}{(c+3)} x+\frac{x^{2}}{(c+2)(c+3)}}$ \\
\hline
\end{tabular}

When $c=\frac{3}{2}$, and $x$ is replaced by $x^{2}$ in (4.1.7), there follows the continued fraction expansion

$$
x e^{-x^{2}}\left\{\frac{1}{1-} \frac{2 x^{2}}{3+} \frac{4 x^{2}}{5-7+} \frac{6 x^{2}}{7+} \cdots\right\}
$$

of the error function 


$$
\operatorname{erf}(x)=\int_{0}^{x} e^{-t^{2}} d t
$$

due to Laplace [19], which was used by Sheppard for the computation of the British Association tables [20] of the error function. When $c=1,(4.1 .7)$ gives the well known expansion of the exponential function

$$
\exp (x)=1+\frac{x}{1-} \frac{x}{2+3-} \frac{x}{4+5-} \frac{2 x}{2 r+} \frac{r x}{2 r+1-} \cdots
$$

and Table 6 is replaced by (numerical values for $x=1$ being appended) Table $6 a$.

TABLE 6a

\begin{tabular}{l|c|c|c|c|l}
\hline 1 & $1+x$ & $1+x+\frac{x^{2}}{2}$ & 1.0 & 2.0 & 2.5 \\
$\frac{1}{1-x}$ & $\frac{1+\frac{x}{2}}{1-\frac{x}{2}}$ & $\frac{1+\frac{2}{3} x+\frac{x^{2}}{12}}{1-\frac{x}{3}}$ & $\infty$ & 3.0 & 2.75 \\
\hline $1-x+\frac{x^{2}}{2}$ & $\frac{1+\frac{x}{3}}{1-\frac{3}{3} x+\frac{x^{2}}{6}}$ & $\frac{1+\frac{x}{2}+\frac{x^{2}}{12}}{1-\frac{x}{2}+\frac{x^{2}}{12}}$ & 2.0 & 2.6 & 2.714286 \\
\hline
\end{tabular}

By replacing $-x^{2}$ by $i x$ in (4.1.8) the Fresnel integrals

(4.1.10) $C(x)=(2 \pi)^{-1 / 2} \int_{0}^{x} t^{-1 / 2} \cos t d t$ and $S(x)=(2 \pi)^{-1 / 2} \int_{0}^{x} t^{-1 / 2} \sin t d t$ may be computed at one blow by evaluating the real and imaginary parts of the expansion

$$
C(x)+i S(x)=\left(\frac{2}{\pi}\right)^{1 / 2} x^{1 / 2}(\cos x+i \sin x)\left\{\frac{1}{1+} \frac{2 i x}{3-} \frac{4 i x}{5+} \frac{6 i x}{7-} \cdots\right\}
$$

Further, by writing $x^{2}$ for $-x^{2}$ in (4.1.8) there follows the expansion of Dawson's integral

$$
\int_{0}^{x} e^{t^{2}} d t=x e^{x^{2}}\left\{\frac{1}{1+3-} \frac{2 x^{2}}{3-4 x^{2}} \frac{6 x^{2}}{7-} \cdots\right\} .
$$

Explicit formulas for the rational approximations corresponding to the functions (3.9.6), (4.1.4) and (4.1.7) are given by Luke [21].

5. Series with Hadamard Gaps. It has been tacitly assumed throughout that none of the Hankel determinants $H_{r}{ }^{(k)}$, defined by equation $(2-4)$, involving the coefficients in the power series

$$
\beta(x)=\sum_{s=0}^{\infty} c_{s} x^{s} \text { and } F(z)=\sum_{s=0}^{\infty} c_{s} z^{-s-1},
$$


vanishes. If this condition is not fulfilled, certain formal difficulties exist in the methods described in this paper, though straight-forward appeal to the set of equations (2.3) may be sufficient to determine certain of the rational quotients. A feature of the Padé table derived for series of this kind is that there are blocks containing the rational functions lying in and upon the rectangle at whose corners stand the quotients

$$
\frac{U_{\mu, \nu}(x)}{V_{\mu, \nu}(x)}, \quad \frac{U_{\mu+m, \nu}(x)}{V_{\mu+m, \nu}(x)}, \quad \frac{U_{\mu, \nu+n}(x)}{V_{\mu, \nu+n}(x)}, \quad \frac{U_{\mu+m, \nu+n}(x)}{V_{\mu+m, \nu+n}(x)}
$$

which, due to cancellation of polynomial factors, contain only the quotient $U_{\mu, \nu}(x) / V_{\mu, \nu}(x)$. The continued fractions whose successive convergents are the distinct quotients lying upon a diagonal in the Padé table are of the form

$$
c_{0}+\frac{a_{1} x^{\alpha_{1}}}{1+} \frac{a_{2} x^{\alpha_{2}}}{1+} \cdots
$$

where $\alpha_{1}, \alpha_{2}, \cdots$ are positive integers. A general theory of such continued fractions and of the resulting rational approximations is not yet available, though Wall [3, p. 399] gives a comprehensive survey of work which has been done upon this problem, and makes a number of interesting conjectures.

It is to be expected that numerical experience obtained in the use of rational approximation formulas will point the way to further theoretical developments.

6. The Efficiency of Various Methods of Obtaining Rational Approximations. The process of obtaining rational approximations to a function formally defined by a power series expansion may be regarded as one solution to the problem of obtaining information about the functions

$$
\beta(x)=\sum_{s=0}^{\infty} c_{s} x^{s} \quad \text { or } \quad F(z)=\sum_{s=0}^{\infty} c_{s} z^{-s-1}
$$

from the finite set of coefficients $c_{s} \quad s=0,1, \cdots, \nu$, where, for convenience in the ensuing discussion, $\nu$ is taken to be $2 n-1$. Numerical experience supports the assertion, as does reference to Tables 3,5 and 7 in the case of particular examples, that in general, of those rational expressions $U_{\mu, \nu}(x) / V_{\mu, \nu}(x)$ or $E_{r}^{(m)}(z)$ which may be obtained from the set of coefficients $c_{s} s=0,1, \cdots, 2 n-1$, (namely those entries in the Padé table lying upon and in the triangle

$$
\frac{U_{0, \nu}(x)}{V_{0, \nu}(x)}, \quad \nu=0,1, \cdots, 2 n-1
$$

$$
\frac{U_{\mu, 0}(x)}{V_{\mu, 0}(x)} \quad \mu=0,1, \cdots, 2 n-1, \quad \frac{U_{2 n-\nu-1, \nu}(x)}{V_{2 n-\nu-1, \nu}(x)} \quad \nu=0,1, \cdots, 2 n-1,
$$

and those entries in the $E$-array which lie in and upon the triangle whose vertices coincide with the functions $\left.E_{0}{ }^{(0)}(z), E_{n}{ }^{(0)}(z), E_{0}{ }^{(2 n)}(z),\right)$ the expressions which contain the most information about the function $\beta(x)$ or $F(z)$, or more precisely, for prescribed $x$ or $z$ the expressions for which

$$
\left|\frac{U_{\mu, \nu}(x)}{V_{\mu, \nu}(x)}-\beta(x)\right| \text { or }\left|F(z)-E_{r}^{(m)}(z)\right|
$$


are a minimum, are given by $\mu+1=\nu=n$ or $\mu=\nu+1=n$, or by $m=0$, $r=n$.

A comparison of the methods devised from results contained in the theoretical part of this paper, which is meaningful in a practical context, may thus be made by contrasting the numbers and types of arithmetic operations needed to compute (versing the discussion in terms of the $E$-array for convenience)

I. the function $\frac{o_{r}^{(0)}(z)}{p_{r}^{(0)}(z)}$

II. the sequence of functions $\frac{o_{r}^{(0)}(z)}{p_{r}^{(0)}(z)} \quad r=1, \cdots, n$

III. the complete array of functions $\frac{r_{r}^{(m)}(z)}{p_{r}^{(m)}(z)} \quad r=1,2, \cdots, n$; $m=0, \cdots, 2 n-2 r$

The remarks contained in sections 2 and 3 indicate that in general any conclusions arrived at relating to methods for the construction of the $E$-array apply with equal force to the construction of the Pade table.

In this comparison, which is about to be made, a further distinction will be drawn between the processes of deriving explicit formulas for the rational functions, and that of computing the values of these functions for some prescribed values of the argument, as would be required, for example, in an initial exploratory study. It is quite clear that in many cases the techniques employed in these two processes will differ, and that considerable saving of computational effort may follow from the foreknowledge that numerical values of the various expressions for a single value of the argument are to be required. For example, assuming $\alpha_{r}^{(m)}, \beta_{r-1}^{(m)}$ to be given, the computation of the coefficients in the polynomial $p_{r+1}^{(m)}(z)$ from the recursion

$$
p_{r+1}^{(m)}(z)=\left(z-\alpha_{r}^{(m)}\right) p_{r}^{(m)}(z)-\beta_{r-1}^{(m)}(z)
$$

requires $2 r-1$ multiplications and $2 r-1$ subtractions, whereas if the polynomials $p_{z}^{(m)}(z)$ are regarded as numerical quantities, the recursion $(6.1)$ involves merely 2 subtractions and 2 multiplications. A distinction of a more subtle kind is indicated by the two recursions

$$
\begin{aligned}
p_{r}^{(m)}(z) & =z p_{r-1}^{(m+1)}-q_{r}^{(m)} p_{r-1}^{(m)}(z) \\
p_{r}^{(m+1)}(z) & =p_{r}^{(m)}(z)-e_{r}^{(m)} p_{r-1}^{(m+1)}(z)
\end{aligned}
$$

For numerical values of $z$, the second is to be preferred to the first for the computation of the polynomials $p_{r}^{(m)}(z)$ since only one multiplication is required (the polynomials $p_{r}^{(0)}(z) \quad r=1,2, \cdots, n$ must, of course, be computed previously to the computation of $p_{r}^{(m)}(z)$, either by use of $(6.1)$ with $m=0$, or by judiciously timed application of $(6.2)$ with $m=0$ ). With regard to the computation of the coefficients in the polynomial however, the first is to be preferred, since multiplication by $z$ corresponds only to the shift of the coefficients vector

$$
\left(k_{r-1, r-1}^{(m+1)}, k_{r-1, r-2}^{(m+1)}, \cdots, k_{r-1,0}^{(m+1)}\right)
$$

and the recursion itself is applicable throughout the whole range of $m$ and $r$ under consideration. 
Any feasible method for constructing the rational functions $E_{r}^{(m)}(z)$ directly from the coefficients $c_{s} s=0,1, \cdots$ may be regarded as proceeding in a number of stages, each of which incorporates a result described earlier in this paper. A systematic study of feasible methods for deriving rational approximations may thus be conducted by describing each of these stages in terms of the numbers and types of arithmetic operations involved and, subsequently, designing the methods by combining suitable steps. The arithmetic operations involved in each method may be assessed by adding together those involved in the composite stages, and a comparison of the methods thus be made.

The following notations will be used:

for the vectors:

$$
\begin{gathered}
\mathbf{p}_{r}^{(m)}=\left(k_{r, r}^{(m)}, k_{r, r-1}^{(m)}, \cdots, k_{r, 0}^{(m)}\right) \\
\mathbf{0}_{r}^{(m)}=\left(j_{r, r-1}^{(m)}, j_{r, r-2}^{(m)}, \cdots, j_{r, 0}^{(m)}\right) \\
\mathbf{r}_{r}^{(m)}=\left(i_{r, m+r-1}^{(m)}, i_{r, m+r-2}^{(m)}, \cdots, i_{r, 0}^{(m)}\right) \\
\mathbf{c}_{r}^{(m)}=\left(c_{m}, c_{m+1}, \cdots, c_{m+r}\right) \\
\mathbf{d}_{t, s}^{(m)}=\left(d_{t, s, s-1}^{(m)}, d_{t, s, s-2}^{(m)}, \cdots, d_{t, s, 0}^{(m)}\right) \\
\mathbf{d}_{t, s}^{(m)}=\left(d_{t, s, s}^{(m)}, d_{t, s, s-1}^{(m)^{\prime}}, \cdots, d_{t, s, 0}^{(m)}\right) \\
\varepsilon_{r}^{(m)}=\left(\epsilon_{r, 2 r}^{(m)}, \epsilon_{r, 2 r-1}^{(m)}, \cdots, \epsilon_{r, 0}^{(m)}\right) \\
\mathbf{z}_{s}^{(m)}=\left(z^{m}, z^{m+1}, \cdots, z^{m+s}\right)
\end{gathered}
$$

for the matrices:

$$
\begin{gathered}
\mathbf{C}_{r}^{(m)}=\left(\begin{array}{cccc}
c_{m} & c_{m+1} & \cdots & c_{m+r} \\
c_{m+1} & c_{m+2} & \cdots & c_{m+r+1} \\
\vdots & \vdots & & \vdots \\
c_{m+r} & c_{m+r+1} & \cdots & c_{m+2 r}
\end{array}\right) \\
\mathbf{H}_{r}^{(m)}=\left(\begin{array}{cccc}
1 & 0 & \cdots & 0 \\
h_{1,0}^{(m)} & 1 & \cdots & 0 \\
\vdots & \vdots & & \vdots \\
h_{r, 0}^{(m)} & h_{r, 1}^{(m)} & \cdots & 1
\end{array}\right) \\
\mathbf{K}_{r}^{(m)}=\left(\begin{array}{cccc}
1 & 0 & \cdots & 0 \\
k_{1,0}^{(m)} & 1 & \cdots & 0 \\
\vdots & \vdots & & \vdots \\
k_{r, 0}^{(m)} & k_{r, 1}^{(m)} & \cdots & 1
\end{array}\right)
\end{gathered}
$$

The matrix

$$
\left(\begin{array}{cccc}
a_{1} & a_{2} & a_{3} & \cdots \\
0 & a_{1} & a_{2} & \cdots \\
0 & 0 & a_{1} & \ldots \\
\ldots & \ldots & \ldots & \ldots
\end{array}\right)
$$

possessing $i$ rows and $j$ columns, derived from the vector $a_{k}=\left(a_{1}, a_{2}, \cdots, a_{k}\right)$ where $a_{s}=0, s>k$, will be denoted by $\left[a_{k}\right]_{i, j}$. The extended vectors $(0, \cdots$. 
$\left.a_{1}, a_{2}, \cdots, a_{k}\right)$ and $\left(a_{1}, a_{2}, \cdots, a_{k}, 0, \cdots, 0\right)$ each possessing $t$ elements, will be denoted by $\left(0, \cdots, 0, a_{k}\right)_{t}$ and $\left(a_{k}, 0, \cdots, 0\right)_{t}$ respectively.

The stages are as follows:

I. Formation of the quantities $q_{r}^{(m)} \quad r=1,2, \cdots, n ; m=0,1, \cdots$, $2 n-2 r, \quad e_{r}^{(m)} \quad r=0,1, \cdots, n-1 ; m=0,1, \cdots, 2 n-2 r-1$, by means of the relations

$$
\begin{aligned}
& e_{0}^{(m)}=0 \quad q_{1}^{(m)}=c_{m+1} / c_{m} \\
& q_{r}^{(m)}+e_{r}^{(m)}=q_{r}^{(m+1)}+e_{r-1}^{(m+1)} \\
& q_{r+1}^{(m)} e_{r}^{(m)}=q_{r}^{(m+1)} e_{r}^{(m+1)}
\end{aligned}
$$

II. Formation of the quantities $\alpha_{r}^{(m)}, \beta_{r-1}^{(m)} m=0, \cdots, 2 n-2 r$ from the relations

$$
\begin{aligned}
& \alpha_{r}^{(m)}=e_{r}^{(m)}+q_{r+1}^{(m)} \quad r=0,1, \cdots, n-1 \\
& \beta_{r}^{(m)}=e_{r+1}^{(m)} q_{r+1}^{(m)} \quad r=0,1, \cdots, n-2
\end{aligned}
$$

III. Generation of the quantities $z^{-1}, z^{-2}, \cdots, z^{-2 n}$

IV. Generation of the quantities $c_{r} z^{-r} \quad r=0, \cdots, 2 n-1$

V. Generation of the quantities $c_{r} z^{-r-1}, \sum_{s=0}^{m-1} c_{s} z^{-s-1}$

$r, m=0, \cdots, 2 n-1$

VI. From the boundary conditions $E_{0}{ }^{(m)}(z)=\sum_{m-1}^{m-1} c_{,} z^{-,-1}$ $m=1, \cdots, 2 n ; E_{0}^{(0)}=0, E_{1 / 2}^{(m)}(z)=z^{m+1} c_{m}{ }^{-1} m=0, \cdots, 2 n-1$, the formation of the quantities $E_{r}^{(m)}(z) \quad m=0, \cdots, 2 n-2 r$ and the quantities $E_{r+1 / 2}^{(m)}(z) \quad m=0, \cdots, 2 n-2 r-1$, by means of the recursions

$$
E_{s+1 / 2}^{(m)}(z)=E_{s-1 / z}^{(m+1)}(z)+\frac{1}{E_{s}^{(m+1)}(z)-E_{s}^{(m)}(z)} \quad s=\frac{1}{2}, 1, \cdots, n-\frac{1}{2}
$$

VII. Generation of the quantities $z-\alpha_{r}{ }^{(0)} r=0, \cdots, n-1$

VIII. Generation of the quantities $z-\alpha_{r}{ }^{(m)}$

$$
m=0, \cdots, 2 n-2 r ; r=0, \cdots, n-1
$$

IX. Generation of the quantities $p_{r}{ }^{(0)}(z)$ from

$$
\begin{aligned}
p_{r+1}^{(0)}(z)= & \left(z-\alpha_{r}{ }^{(0)}\right) p_{r}{ }^{(0)}(z)-\beta_{r-1}^{(0)} p_{r-1}^{(0)}(z) \\
r & =1, \cdots, n-1, \quad p_{0}{ }^{(0)}(z)=1, \quad p_{1}{ }^{(0)}(z)=z-q_{1}{ }^{(0)} .
\end{aligned}
$$

$X$ : Generation of the quantities $o_{r}^{(0)}(z)$ from

$$
\begin{aligned}
& o_{r+1}^{(0)}(z)=\left(z-\alpha_{r}^{(0)}\right) o_{r}^{(0)}(z)-\beta_{r-1}^{(0)} o_{r-1}^{(0)}(z) \\
& \quad r=1, \cdots, n-1, \quad o_{0}^{(0)}(z)=0, \quad 0_{1}{ }^{(0)}=c_{0}
\end{aligned}
$$

XI. Generation of the quantities $p_{r}^{(0)}(z) \quad r=2, \cdots, n$ from

$$
p_{r}{ }^{(0)}(z)=z p_{r-1}^{(1)}(z)-q_{r}{ }^{(0)} p_{r-1}^{(0)}(z)
$$

XII. Generation of the quantities $p_{r}{ }^{(m+1)}(z)$ from

$$
\begin{aligned}
p_{r}^{(m+1)}(z)=p_{r}^{(m)}(z) & -e_{r}^{(m)} p_{r-1}^{(m+1)}(z) \\
r & =1, \cdots, n-1 ; \quad m=0, \cdots, 2 n-2 r-1
\end{aligned}
$$


XIII. Generation of the quantities $r_{r}^{(0)}(z)$ from

$$
r_{r}^{(0)}(z)=z r_{r-1}^{(1)}(z)-q_{r}^{(0)} r_{r-1}^{(0)}(z) \quad r=2, \cdots, n
$$

XIV. Generation of the quantities $r_{r}^{(m+1)}(z)$ from

$$
\begin{aligned}
r_{r}^{(m+1)}(z)=r_{r}^{(m)}(z) & -e_{r}^{(m)} r_{r-1}^{(m+1)}(z) \\
r & =1, \cdots, n-1 ; \quad n=0, \cdots, 2 n-2 r-1
\end{aligned}
$$

XV. The single division $o_{n}^{(0)}(z) / p_{n}{ }^{(0)}(z)$.

XVI. The divisions $o_{r}{ }^{(0)}(z) / p_{r}{ }^{(0)}(z) \quad r=1, \cdots, n$

XVII. The divisions $r_{r}^{(m)}(z) / p_{r}^{(m)}(z) \quad r=1, \cdots, n ; m=0, \cdots, 2 n-2 r$

XVIII. Computation of $\frac{0_{r}^{(0)}(z)}{p_{r}{ }^{(0)}(z)}=\frac{a_{1}}{b_{1} t} \cdots \frac{a_{r}}{b_{r}}$, where $a_{1}=c_{0} ; a_{s}=-\beta_{s-2}{ }^{(0)}$; $s=2, \cdots, r ; b_{s+1}=z-\alpha_{s}{ }^{(0)} ; s=0, \cdots, r-1$, from

$$
D_{1}=\frac{a_{r}}{b_{r}}, \quad D_{r+1}=\frac{a_{r-s}}{b_{r-s}+D_{s}}, \quad D_{r}=\frac{o_{r}^{(0)}(z)}{p_{r}^{(0)}(z)}
$$

when $r=n$.

XIX. The use of XVIII. when $r=1, \cdots, n$

$\mathrm{XX}$. Computation of

$$
\begin{aligned}
\frac{r_{r}^{(m)}(z)}{p_{r}^{(m)}(z)} & =\sum_{s=0}^{m-1} c_{s} z^{-s-1}+\frac{c_{m} z^{-m}}{z-\alpha_{0}^{(m)}-} \frac{\beta_{0}^{(m)}}{z-\alpha_{1}^{(m)}-} \cdots \frac{\beta_{r-2}^{(m)}}{z-\alpha_{r-1}^{(m)}} \\
& =b_{0}+\frac{a_{1}}{b_{1}+} \cdots \frac{a_{r}}{b_{r}}
\end{aligned}
$$

where $b_{0}=\sum_{s=0}^{m-1} c_{s} z^{-s-1} ; a_{1}=c_{m} z^{-m} ; a_{s}=\beta_{s-2}^{(m)} ; s=2, \cdots, r$;

$b_{s}=z-\alpha_{s-1}^{(m)} ; s=1, \cdots, r$ by

$$
D_{1}=b_{r}, \quad D_{s+1}=b_{r-s}+\frac{a_{r-s+1}}{D_{s}}, \quad D_{r+1}=\frac{r_{r}^{(m)}(z)}{p_{r}^{(m)}(z)}
$$

for $r=1, \cdots, n-1 ; m=1, \cdots, 2 n-2 r$.

XXI. Computation of the products $c_{0} \beta_{0}{ }^{(0)} \cdots \beta_{r-1}^{(0)} \quad r=1, \cdots, n-1$.

XXII. Computation of the products $z^{-m} c_{m} \beta_{0}{ }^{(m)} \cdots \beta_{r-1}^{(m)} \quad r=1, \cdots, n-1$; $m=1, \cdots, 2 n-2 r$.

XXIII. Computation of the convergents

$$
\frac{o_{r}{ }^{(0)}(z)}{p_{r}{ }^{(0)}(z)}=\sum_{s=1}^{s-r} \frac{c_{0} \beta_{0}{ }^{(0)} \cdots \beta_{s-2}^{(0)}}{p_{s-1}^{(0)}(z) p_{s}{ }^{(0)}(z)} \quad r=1, \cdots, n
$$

XXIV. Computation of the convergents

$$
\frac{r_{r}{ }^{(m)}(z)}{p_{r}{ }^{(m)}(z)}=\sum_{s=0}^{m-1} c_{s} z^{-s-1}+\sum_{s=1}^{s=r} z^{-m} \frac{c_{m} \beta_{0}{ }^{(m)} \cdots \beta_{s-2}^{(m)}}{p_{s-1}^{(m)}(z) p_{s}{ }^{(m)}(z)} \quad \begin{array}{ll}
r=1, \cdots, n-1 \\
m=1, \cdots, 2 n-2 r
\end{array}
$$

XXV. Computation of $p_{r}{ }^{(m)}$ from $p_{0}{ }^{(m)}=(1) ; p_{1}{ }^{(m)}=\left(1,-c_{m+1} / c_{m}\right)$; $p_{r+1}^{(m)}=\left(p_{r}^{(m)}, 0\right)_{r+2}-\alpha_{r}^{(m)}\left(0, p_{r}^{(m)}\right)_{r+2}-\beta_{r-1}^{(m)}\left(0,0, p_{r-1}^{(m)}\right)_{r+2}$ $r=1, \cdots, n-1$

for $m=0$

$$
\begin{aligned}
& \alpha_{r}{ }^{(m)}=\frac{\mathbf{p}_{r}{ }^{(m)} \mathbf{c}_{r}{ }^{(m+r+1) T}}{\mathbf{p}_{r-1}^{(m)} \mathbf{c}_{r-1}^{(m+r+1) T}}-\frac{\mathbf{p}_{r-1}^{(m)} \mathbf{c}_{r-1}^{(m+r+1) T}}{\mathbf{p}_{r-2}^{(m)} \mathbf{c}_{r-2}^{(m+r+1) T}} \\
& \beta_{r-1}^{(m)}=\frac{\mathbf{p}_{r}{ }^{(m)} \mathbf{c}_{r}{ }^{(m+r+1) T}}{\mathbf{p}_{r-1}^{(m)} \mathbf{c}_{r-1}^{(m+r+1) T}} \quad r=1, \cdots, n-1
\end{aligned}
$$


XXVI. XXV for $m=0,2, \cdots, 2 n-2 ; r=1,2, \cdots, n-(m / 2): m=1,3, \cdots$, $2 n-3 ; r=1,2, \cdots, n-(m+1) / 2$

XXVII. Computation of $o_{r}{ }^{(0)}$ from $o_{0}^{(0)}=0 ; o_{1}{ }^{(0)}=\left(c_{0}\right)$

$$
\begin{aligned}
0_{r+1}^{(0)}=\left(0_{r}^{(0)}, 0\right)_{r+1}-\alpha_{r}^{(0)}\left(0,0_{r}^{(0)}\right)_{r+1}-\beta_{r-1}^{(0)}\left(0,0,0_{r-1}^{(0)}\right)_{r+1} & \\
r & =1, \cdots, n-1
\end{aligned}
$$

XXVIII. Computation of $p_{r}{ }^{(0)} \quad r=1, \cdots, n$ from $p_{0}{ }^{(0)}=(1) ; p_{1}{ }^{(0)}=\left(1,-c_{1} / c_{0}\right)$

$$
p_{r+1}^{(0)}=\left(p_{r}^{(0)}, 0\right)_{r+2}-\alpha_{r}^{(0)}\left(0, p_{r}^{(0)}\right)_{r+2}-\beta_{r-1}^{(0)}\left(0,0, p_{r-1}^{(0)}\right)_{r+2}
$$

XXIX. Gauss-Banachiewicz decomposition of the matrix $\mathbf{C}_{n}{ }^{(0)}$

$$
\mathbf{C}_{n}{ }^{(0)}=\mathbf{H}_{n}{ }^{(0)} \mathbf{D}_{n}{ }^{(0)} \mathbf{H}_{n}{ }^{(0) T}
$$

XXX. Decomposition of the matrices $\mathbf{C}_{r}^{(m)} m=0, \cdots, 2 n-4$;

$r=n-2 m, n-2 m+1$

XXXI. Recovery of the quantities $\beta_{r-1}^{(0)} r=1, \cdots, n-1$ from $\mathrm{D}_{n}{ }^{(0)}$ by means of the relations

$$
\beta_{r-1}^{(0)}=\frac{c_{0} \beta_{0}^{(0)} \cdots \beta_{r-1}^{(0)}}{c_{0} \beta_{0}{ }^{(0)} \cdots \beta_{r-2}^{(0)}}
$$

XXXII. Recovery of the quantities $\alpha_{r}{ }^{(0)} \quad r=0, \ldots, n-1$ from $\mathrm{H}_{n}{ }^{(0)}$ by means of the relations

$$
\alpha_{r}^{(0)}=h_{r, r-1}^{(0)}-h_{r+1, r}^{(0)} \quad \alpha_{0}^{(0)}=-h_{1,0}^{(0)}
$$

XXXIII. Computation of the quantities $\alpha_{r}^{(m)} \quad r=0, \cdots, n-1 ; m=0, \cdots$, $2 n-2 r ; \beta_{r}{ }^{(m)} \quad r=0, \cdots, n-2 ; m=0, \cdots, 2 n-2 r-1$ from $\mathbf{H}_{r}{ }^{(m)}$ and $D_{r}{ }^{(m)}$ by means of the relationships

$$
\alpha_{r}^{(m)}=h_{r, r-1}^{(m)}-h_{r+1, r}^{(m)} \quad \beta_{r-1}^{(m)}=\frac{c_{m} \beta_{0}^{(m)} \cdots \beta_{r-1}^{(m)}}{c_{m} \beta_{0}^{(m)} \cdots \beta_{r-2}^{(m)}}
$$

XXXIV. Computation of the quantities $q_{r}{ }^{(m)} \quad r=1, \cdots, n ; m=0, \cdots$, $2 n-2 r ; \quad e_{r}^{(m)} \quad r=1, \cdots, n-1 ; m=0, \cdots, 2 n-2 r-1$ from

$$
q_{r+1}^{(m)}=\alpha_{r}^{(m)}-e_{r}^{(m)} \quad e_{r}^{(m)}=\beta_{r-1}^{(m)} / q_{r}{ }^{(m)}
$$

XXXV. Computation of the matrix $\mathbf{K}_{n}{ }^{(0)}$ by inversion of $\mathbf{H}_{n}{ }^{(0)}$

$$
\mathbf{H}_{n}{ }^{(0)} \mathbf{K}_{n}{ }^{(0)}=I
$$

XXXVI. Computation of the matrices $\mathbf{K}_{r}{ }^{(m)}$ by inversion of the matrices $\mathbf{H}_{r}{ }^{(m)}$

$$
\mathbf{H}_{r}{ }^{(m)} \mathbf{K}_{r}{ }^{(m)}=\mathbf{I} \quad r=2, \cdots, n ; m=2 n-2 r, 2 n-2 r-1
$$

in conjunction with the independent formation of $p_{1}{ }^{(2 n-2)}$ and $p_{1}{ }^{(2 n-3)}$

XXXVII. Computation of the quantities $q_{r}^{(m)} r=1, \cdots, n ; m=0, \cdots$, $2 n-2 r$ from the relations

$$
q_{r}^{(m)}=k_{r-1, r-2}^{(m)}-k_{r, r-1}^{(m)}
$$

XXXVIII. Computation of $\mathrm{p}_{r}^{(m)} \quad r=1, \cdots, n ; m=0, \cdots, 2 n-2 r$ from the recursion

$$
p_{r}{ }^{(m)}=\left(p_{r-1}^{(m+1)}, 0\right)_{r+1}-q_{r}{ }^{(m)}\left(0, p_{r-1}^{(m)}\right)_{r+1} \quad p_{1}{ }^{(m)}=\left(1,-q_{1}{ }^{(m)}\right)
$$


XXXIX. Computation of $r_{r}^{(m)} \quad r=1, \cdots, n ; m=0, \cdots, 2 n-2 r$ from the recursion

$$
\begin{aligned}
r_{r}{ }^{(m)}=r_{r-1}^{(m+1)}-q_{r}{ }^{(m)} & \left(0, r_{r-1}^{(m)}\right)_{r+m-1} \\
& r_{0}{ }^{(m)}=c_{m-1}^{(0)} \quad m=1, \cdots, 2 n-2 r ; r_{0}{ }^{(0)}=0
\end{aligned}
$$

XL. Computation of $o_{r}{ }^{(m)} \quad r=1, \cdots, n ; m=0, \cdots, 2 n-2 r$ from the recursion

$$
0_{r}{ }^{(m)}=c_{m} \mathrm{p}_{r-1}^{(m+1)}+\left(0,0_{r-1}^{(m+1)}\right)_{r}-q_{r}{ }^{(m)}\left(0,0_{r-1}^{(m)}\right)_{r} \quad 0_{1}{ }^{(m)}=c_{m}
$$

XLI. Computation of $r_{r}{ }^{(m)} \quad r=1, \cdots, n ; m=0, \cdots, 2 n-2 r$ from

$$
r_{r}{ }^{(m)}=p_{r}{ }^{(m)}\left[C_{m-1}^{(0)}\right]_{r+1, m+r}+\left(0, \cdots, 0, \mathbf{O}_{r}{ }^{(m)}\right)_{m+r}
$$

XIII. Computation of $\boldsymbol{o}_{n}{ }^{(0)}$ from

$$
\boldsymbol{O}_{n}{ }^{(0)}=\mathbf{p}_{n}^{(0)}\left[\mathbf{c}_{n-1}^{(0)}\right]_{n+1, n}
$$

XLIII. Computation of $o_{r}{ }^{(0)} \quad r=1, \cdots, n$ from

$$
\mathbf{o}_{r}{ }^{(0)}=\mathrm{p}_{r}{ }^{(0)}\left[\mathrm{c}_{r-1}^{(0)}\right]_{r+1 . r}
$$

XLIV. Computation of $r_{r}^{(m)} \quad r=1, \cdots, n-1 ; m=1, \cdots, 2 n-2 r$ from

$$
\mathbf{r}_{r}^{(m)}=\operatorname{pr}^{(m)}\left[\mathbf{C}_{r+m-1}^{(0)}\right]_{r+1, m+r}
$$

XLV. Computation of $\mathbf{r}_{r}{ }^{(0)} \quad r=1, \cdots, n$ from

$$
\mathbf{p r}^{(0)}\left[\mathbf{r}_{r-1}^{(0)}\right]_{r+1, r}=\mathbf{p}_{r-1}^{(0)}\left[\mathbf{r}_{r}{ }^{(0)}\right]_{r, r}
$$

XLVI. Determination of $r_{r}^{(m)} \quad r=1, \cdots, n-1 ; m=1, \cdots, 2 n-2 r$ from

$$
\mathbf{p}_{r}{ }^{(m)}\left[\mathbf{r}_{r-1}^{(m)}\right]_{r+1, m+r}=\mathbf{p}_{r-1}^{(m)}\left[\mathbf{r}_{r}{ }^{(m)}\right]_{r, m+r}
$$

XLVII. The determination of the vectors $d_{n, n}^{(0)}=0_{n}{ }^{(0)}, d_{n, n}^{(0)}=p_{n}{ }^{(0)}$ from the recursions

$$
\begin{gathered}
\mathrm{d}_{n, 1}^{(0)}=\left(\beta_{n-2}^{(0)}\right) \quad \mathrm{d}_{n, 1}^{(0)^{\prime}}=\left(1,-\alpha_{n-1}^{(0)}\right) \quad \mathrm{d}_{n, s}^{(0)}=\beta_{n-s-1}^{(0)} \mathrm{d}_{n, s-1}^{(0)^{\prime}}, \\
\mathrm{d}_{n, s}^{(0)^{\prime}}=\left(\mathrm{d}_{n, s-1}^{(0)^{\prime}}, 0\right)_{s+1}-\alpha_{n-s}^{(0)}\left(0, \mathrm{~d}_{n, s-1}^{(0)^{\prime}}\right)_{s+1}+\left(0,0, \mathrm{~d}_{n, s-1}^{(0)}\right)_{s+1} \\
s=2, \cdots, n ; \beta_{-1}^{(0)}=c_{0}
\end{gathered}
$$

XLVIII. The determination of the vectors $\mathbf{d}_{n-r, n}^{(0)}=\mathbf{o}_{n-r}^{(0)}, \mathbf{d}_{n-r, n}^{(0)}=\mathbf{p}_{n-r}^{(0)}$ $r=0, \cdots, n-1$ from the recursions

$$
\begin{array}{r}
\mathbf{d}_{r, r}^{(0)}=\left(\beta_{n-r-2}^{(0)}\right) \quad \mathrm{d}_{r, r}^{(0)^{\prime}}=\left(1,-\alpha_{n-r-1}^{(0)}\right) \quad \mathrm{d}_{r, r+s}^{(0)}=\beta_{n \rightarrow-s-2}^{(0)} \mathbf{d}_{r, r+s-1}^{(0)^{\prime}}, \\
\mathbf{d}_{r, r+b}^{(0)^{\prime}}=\left(\mathbf{d}_{r, r+s-1}^{(0)^{\prime}}, 0\right)_{s+2}-\alpha_{n-r-s-1}^{(0)}\left(0, \mathbf{d}_{r, r+s-1}^{(0)}\right)_{s+2}^{\prime}+\left(0,0, \mathbf{d}_{r, r+s-1}^{(0)}\right)_{s+2} \\
r=0, \cdots, n-1 ; s=1, \cdots, n-r ; \beta_{-1}^{(0)}=c_{0}
\end{array}
$$

XLIX. The determination of the vectors $d_{r, \nu}^{(m)}=o_{r}{ }^{(m)}, d_{r, \nu}^{(m)^{\prime}}=p_{r}{ }^{(m)}$ from the recursions

$$
\begin{aligned}
& \mathbf{d}_{r, r}^{(m)}=\left(\beta_{v-r-2}^{(m)}\right) \quad \mathbf{d}_{r, r}^{(m)^{\prime}}=\left(1,-\alpha_{v-r-1}^{(m)}\right) \quad \mathbf{d}_{r, r+o}^{(m)}=\beta_{v-r-s-2}^{(m)} \mathbf{d}_{r, r+\varepsilon-1}^{(m)^{\prime}}, \\
& \mathrm{d}_{r, r+s}^{(m)^{\prime}}=\left(d_{r, r+s-1}^{(m)^{\prime}}, 0\right)_{s+2}-\alpha_{v-r-8-1}^{(m)}\left(0, d_{r, r+s-1}^{(m)}\right)_{s+2}^{\prime}+\left(0,0, d_{r, r+s-1}^{(m)}\right)_{\ell+2} \\
& \beta_{-1}^{(m)}=c_{m}
\end{aligned}
$$




$$
\begin{array}{lll}
m=0,2, \cdots, 2 n-2, & \nu=n-\frac{m}{2} & r=0, \cdots, \nu-1, \\
m=1,3, \cdots, 2 n-1, & \nu=n-\frac{(m+1)}{2} & r=0, \cdots, \nu-1, \\
& s=1, \cdots, \nu-r
\end{array}
$$

L. Determination of $p_{r+1}^{(m)} r=0, \cdots, n-1 ; m=0, \cdots, 2 n-2 r-2$, from

$$
\mathbf{p}_{r-1}^{(m+1)}\left[p_{r+1}^{(m)}\right]_{r, r+2}=\left(\epsilon_{r, 2 r}^{(m+1)}\right)^{-1}\left\{\left(\varepsilon_{r}^{(m+1)}, 0\right)_{r+3}-\left(0, \varepsilon_{r}^{(m)}\right)_{r+3}\right\}
$$

and of $\varepsilon_{r}^{(m)} \quad r=0, \cdots, n-1 ; m=0, \cdots, 2 n-2 r-1$ from

$$
\varepsilon_{r}^{(m)}=\left(0, \varepsilon_{r-1}^{(m+1)}, 0\right)_{2 r+1}+\left(k_{r, 0}^{(m)} i_{r, 0}^{(m+1)}\right)^{-1} p_{r}^{(m+1)}\left[p_{r}^{(m)}\right]_{r+1,2 r+1}
$$

with $\mathrm{p}_{1}{ }^{(m)}=\left(1, c_{m+1} / c_{m}\right), \varepsilon_{0}{ }^{(m)}=\left(c_{m-1}\right)$.

LI. Construction of the vector $z_{n}{ }^{(0)}$

LII. Computation of $p_{n}{ }^{(0)}(z)$ from $p_{n}{ }^{(0)}(z)=p_{n}{ }^{(0)} z_{n}{ }^{(0)} T$

LIII. Computation of $o_{n}{ }^{(0)}(z)$ from $o_{n}{ }^{(0)}(z)=0_{n}{ }^{(0)} z_{n-1}^{(0)} r$

LIV. Computation of $p_{r}{ }^{(0)}(z) \quad r=1, \cdots, n$ from $p_{r}{ }^{(0)}(z)=p_{r}{ }^{(0)} z_{r}{ }^{(0)} T$

LV. Computation of $o_{r}{ }^{(0)}(z) \quad r=1, \cdots, n$ from $o_{r}^{(0)}(z)=0_{r}^{(0)} z_{r}^{(0)}$

LVI. Construction of the vector $z_{3 n}^{(-n)}$

LVII. Computation of $p_{r}^{(m)}(z) \quad r=1, \cdots, n ; m=0, \cdots, 2 n-2 r$ from $p_{r}{ }^{(m)}(z)=p_{r}{ }^{(m)} z_{r}{ }^{(0)} r$

LVIII. Computation of $r_{r}^{(m)}(z) \quad r=1, \cdots, n ; m=0, \cdots, 2 n-2 r$ from

\begin{tabular}{|c|c|c|c|c|c|}
\hline & Multiplication & Division & Addition & Subtraction & Add. + Sub. \\
\hline 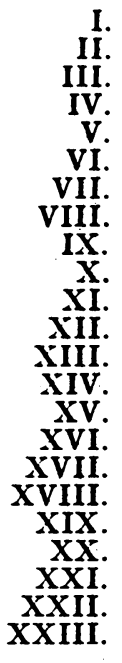 & $\begin{array}{l}2 n-3 \\
2 n-3 \\
2 n-2 \\
n^{2}-3 n+2 \\
2 n-2 \\
n^{2}-n\end{array}$ & $\begin{array}{l}1 \\
n \\
2 n^{2}-n \\
n-1 \\
\frac{1}{2}\left(n^{2}-n\right) \\
\frac{1}{3}\left(n^{3}-n\right) \\
n\end{array}$ & $\begin{array}{l}n \\
n\left(n^{2}+n\right) \\
\frac{1}{3}\left(n^{3}-n\right) \\
n-1\end{array}$ & $\begin{array}{l}n^{2}-n \\
\\
2 n^{2}-n \\
n \\
n^{2} \\
n-1 \\
n-2 \\
n-2 \\
n^{2}-n+1 \\
n-1 \\
n^{2}-n\end{array}$ & $\begin{array}{l}2 n^{2}-4 n+2 \\
n-1 \\
2 n-1 \\
4 n^{2}-2 n \\
n \\
n^{2} \\
n-1 \\
n-2 \\
n-2 \\
n^{2}-n+1 \\
n-1 \\
n^{2}-n \\
\\
n \\
n\left(n^{2}+n\right) \\
\frac{1}{3}\left(n^{3}-n\right) \\
n-1\end{array}$ \\
\hline
\end{tabular}
$r_{r}^{(m)}(z)=r_{r}^{(m)} z_{m+r-1}^{(-m) r}$

The types and numbers of arithmetic operations required in the performance of each of the stages described are given in Table 7.

TABLE 7 
TABLE 7-Continued

\begin{tabular}{|c|c|c|c|c|c|}
\hline & Iultiplication & n & ddition & tion & - Sub. \\
\hline $\begin{array}{r}\text { XXIV. } \\
\text { XXV. } \\
\text { XXVI. } \\
\text { XXVII. } \\
\text { XXVIII. } \\
\text { XXIX. } \\
\text { XXX. } \\
\text { XXXI. } \\
\text { XXXII. } \\
\text { XXXII. } \\
\text { XXXIV. } \\
\text { XXXV. } \\
\text { XXXVI. } \\
\text { XXXVII. } \\
\text { XXXVII. } \\
\text { XXXIX. } \\
\text { XI. } \\
\text { XLI. } \\
\text { XLII. } \\
\text { XLIII. } \\
\text { XLIV. } \\
\text { XLV. } \\
\text { XLVI. }\end{array}$ & 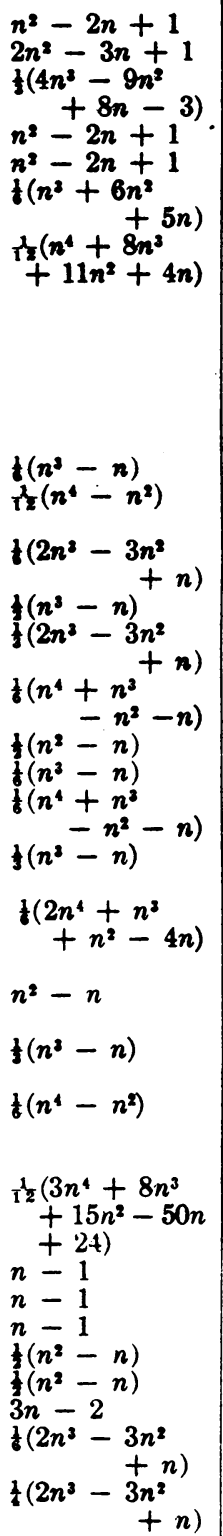 & 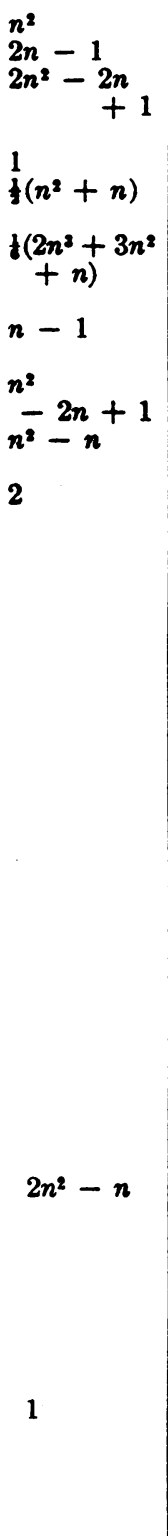 & 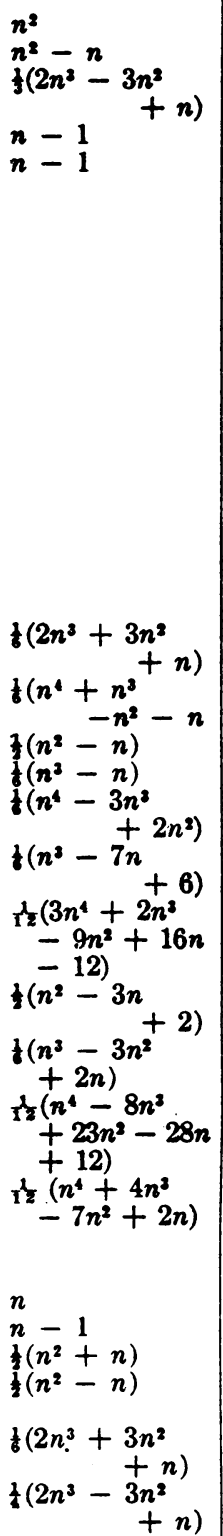 & 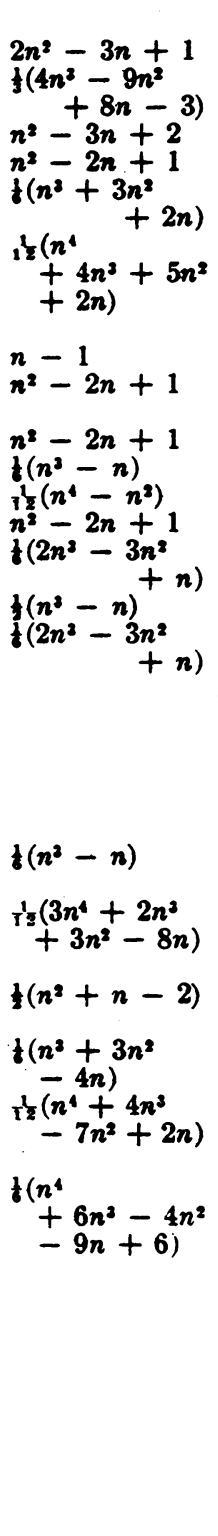 & 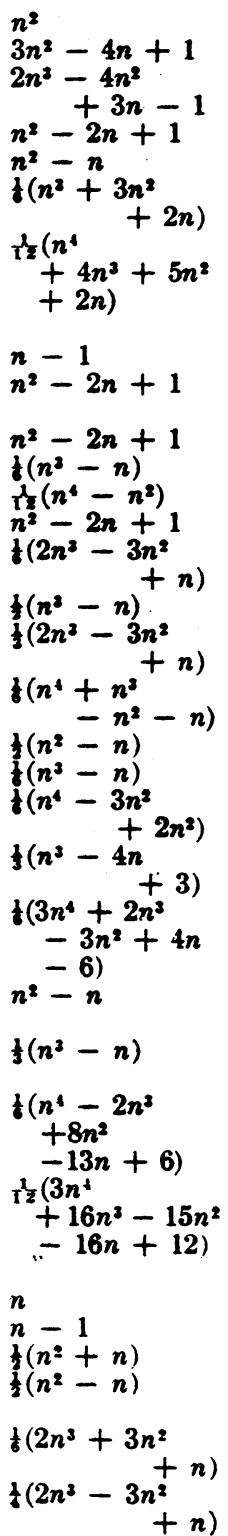 \\
\hline
\end{tabular}

Since addition and subtraction may be performed with quantities of either sign, the distinction between these two operations is somewhat artificial, and in this instance a column denoting the joint number of additions and subtractions has been added to Table 7. It is assumed that multiplications by zero or unity do not occur, and that divisions in which the quotient or divisor is unity also do not occur. The sign reversal of a number is also not taken into account. It is further assumed that 
each of the computations takes place in the most efficient manner possible. For example it is assumed that the quantities

$$
\begin{aligned}
\alpha_{r}{ }^{(m)}=e_{r}^{(m)}+q_{r+1}^{(m)} \quad \beta_{r}^{(m)}=e_{r+1}^{(m)} q_{r+1}^{(m)} & \\
r & =0, \cdots, n-1 ; m=1, \cdots, 2 n-2 r
\end{aligned}
$$

are computed in the process of forming the quantities $e_{r}^{(m)} r=1, \cdots, n-1$; $m=0, \cdots, 2 n-2 r-1, q_{r}^{(m)} \quad r=1, \cdots, n ; m=0, \cdots, 2 n-2 r$ by means of the $q-d$ algorithm relationships

$$
q_{r}^{(m)}+e_{r}^{(m)}=q_{r}^{(m+1)}+e_{r-1}^{(m+1)} \quad q_{r-1}^{(m)} e_{r}^{(m)}=q_{r}^{(m+1)} e_{r}^{(m+1)}
$$

so that the formation of the quantities $\alpha_{r}{ }^{(m)} \quad r=0, \cdots, n-1 \beta_{r}^{(m)} \quad r=0$, $\cdots, n-2 ; m=0, \cdots, 2 n-2 r-4$ from the quantities $e_{r}{ }^{(m)}, q_{r}{ }^{(m)}$ requires only a further $n-1$ additions and $n-1$ multiplications.

Formulas, the use of which would clearly result in inferior computational practice without the existence of compensatory factors, have not been examined. For example the vectors $r_{r}{ }^{(m)} \quad r=1 \cdots, n ; m=0, \cdots, 2 n-2 r$ should clearly be derived from the recursion

$$
\mathbf{r}_{r}^{(m)}=\mathbf{r}_{r-1}^{(m+1)}-q_{r}^{(m)}\left(0, \mathbf{r}_{r-1}^{(m)}\right)_{r+m-1}
$$

rather than from the recursion

$$
\mathbf{r}_{r+1}^{(m)}=\left(\mathbf{r}_{r}^{(m)}, 0\right)_{m+r}-\alpha_{r}^{(m)}\left(0, \mathbf{r}_{r}^{(m)}\right)_{m+r}-\beta_{r-1}^{(m)}\left(0,0, \mathbf{r}_{r-1}^{(m)}\right)_{m+r}
$$

since the first involves only one scalar multiplication and the second two. If the quantities $\dot{\alpha}_{r}^{(m)}, \beta_{r-1}^{(m)}$ are available the quantities $q_{r}^{(m)}$ may recursively be determined from them (viz. XXXIV) and if the vectors $p_{r}{ }^{(m)}$ are already to hand, the quantities $q_{r}{ }^{(m)}$ may be derived by (XXXVII).

Feasible methods of obtaining rational approximations, together with the number and type of arithmetic operators involved, now follow (Tables 8-13).

\section{TAble 8}

Single Value $E_{n}^{(0)}(z)$

A: III, V, VI;

\begin{tabular}{|c|c|c|c|c|c|}
\hline & Multiplications & Divisions & Additions & Subtractions & Add. + Subs. \\
\hline $\begin{array}{l}\mathbf{A} \\
\mathbf{B} \\
\mathbf{C} \\
\mathbf{D} \\
\mathbf{E}\end{array}$ & $\begin{array}{l}4 n-1 \\
n^{2}-n \\
n^{2}+n-3 \\
n^{2}+n-2 \\
+\left(2 n^{3}+9 n^{2}\right. \\
+19 n-18)\end{array}$ & $\begin{array}{l}2 n^{2}+n+1 \\
n^{2}-n-1 \\
n^{2}+1 \\
n^{2}+n \\
\frac{1}{2}\left(n^{2}+n+2\right)\end{array}$ & $\begin{array}{l}2 n^{2}+n-1 \\
n^{2}-n+1 \\
n^{2}-2 n+1 \\
n^{2}-n \\
f\left(n^{3}+6 n^{2}-n\right)\end{array}$ & $\begin{array}{l}2 n^{2}-n \\
n^{2} \\
n^{2}+2 n-3 \\
n^{2}+n-1 \\
\quad \frac{1}{6}\left(2 n^{3}\right. \\
\left.\quad+3 n^{2}+n\right)\end{array}$ & $\begin{array}{l}4 n^{2}-1 \\
2 n^{2}-n+1 \\
2 n^{2}-2 \\
2 n^{2}-1 \\
\frac{1}{2}\left(n^{3}+3 n^{2}+5 n-3\right) \\
\quad+5 n-3\end{array}$ \\
\hline
\end{tabular}

B: I, II, VII, XVIII;

C: I, II, VII, IX, $\mathbf{X}, \mathbf{X V}$;

D: I, II, VII, IX, XXI, XXIII,

E: XXIX, XXXV, XLII, LI, LII, LIII, XV: 
TABLE 9

Diagonal of Values $E_{r}^{(0)}(z) \quad r=1, \cdots, n$

A:

F: I, II, VII, XIX;

G: I, II, VII, IX, X, XVI;

D:

H: XXIX, XXXV, XLIII, LI, LIV, LV, XVI:

\begin{tabular}{|c|c|c|c|c|c|}
\hline & Multiplications & Divisions & Additions & Subtractions & Adds. + Subs. \\
\hline $\begin{array}{l}\mathbf{A} \\
\mathbf{F} \\
\mathbf{G} \\
\mathbf{D} \\
\mathbf{H}\end{array}$ & $\begin{array}{l}4 n-1 \\
n^{2}-n \\
n^{2}+3 n-6 \\
n^{2}+3 n-5 \\
\frac{1}{3}+4 n^{2}+n \\
\quad-2)\end{array}$ & $\begin{array}{l}2 n^{2}+n+1 \\
\frac{1}{2}\left(3 n^{2}-1\right) \\
n^{2}+n \\
n^{2}+n \\
\frac{1}{2}\left(n^{2}+3 n\right)\end{array}$ & $\begin{array}{l}2 n^{2}+n-1 \\
\frac{1}{2}\left(3 n^{2}-3 n+2\right) \\
n^{2}-2 n+1 \\
n^{2}-n \\
6\left(n^{2}+6 n^{2}-n\right)\end{array}$ & $\begin{array}{l}2 n^{2}-n \\
n^{2} \\
n^{2}+2 n-3 \\
n^{2}+n-1 \\
\frac{1}{6}\left(n^{3}+3 n^{2}+n\right)\end{array}$ & $\begin{array}{l}4 n^{2}-1 \\
\frac{1}{3}\left(5 n^{2}-3 n+2\right) \\
2 n^{2}-2 \\
2 n^{2}-1 \\
\frac{1}{3}\left(n^{3}+3 n^{2}\right)\end{array}$ \\
\hline
\end{tabular}

\section{TABLE 10}

Array of Values $\mathrm{E}_{r}^{(m)}(z) \quad r=0, \cdots, n ; m=0, \cdots, 2 n-2 r$

A.

I: I, II, IV, V, VIII, XX;

J: I, III, V, VIII, XI, XII, XXI, XXII, XXIII, XXIV;

K: I, III, V, XI, XII, XIII, XIV, XVII;

L: XXX, XXXVI, XIIV, LVI, LVII, LVIII, XVII;

\begin{tabular}{|c|c|c|c|c|c|}
\hline & Multiplications & Divisions & Additions & Subtractions & Adds. + Subs. \\
\hline $\begin{array}{l}\mathbf{J} \\
\mathbf{K} \\
\mathbf{L}\end{array}$ & $\begin{array}{l}4 n-1 \\
n^{2}+2 n-1 \\
3 n^{2}-2 n+1 \\
3 n^{2}+2 n-2 \\
\quad+2\left(4 n^{4}\right. \\
\quad+20 n^{3}+5 n^{2} \\
\quad+67 n-24)\end{array}$ & $\begin{array}{l}2 n^{2}+n+1 \\
\frac{1}{3}\left(n^{3}+3 n^{2}\right. \\
\quad-n+3) \\
2 n^{2}+n+1 \\
3 n^{2}-n+1 \\
\frac{1}{6}\left(2 n^{3}+21 n^{2}\right. \\
\quad-5 n+24)\end{array}$ & $\begin{array}{l}2 n^{2}+n-1 \\
\frac{1}{3}\left(n^{3}+3 n^{2}-n\right) \\
2 n^{2}-n+1 \\
n^{2}-n+2 n^{4} \\
\quad \frac{1}{2}\left(2 n^{3}+13 n^{2}\right. \\
\quad+4 n+24)\end{array}$ & $\begin{array}{l}2 n^{2}-n \\
2 n^{2}-n \\
3 n^{2}-n-1 \\
3 n^{2}-n-2 \\
\frac{1}{6}\left(n^{4}\right. \\
\quad+2 n^{2}+8 n^{2} \\
\quad-5 n)\end{array}$ & $\begin{array}{l}4 n^{2}-1 \\
\frac{1}{3}\left(n^{3}+9 n^{2}\right. \\
\left.5 n^{2}-n-4 n\right) \\
4 n^{2}-2 n-1 \\
\quad \frac{1}{6}\left(4 n^{4}\right. \\
\quad+8 n^{2}+29 n^{2} \\
\quad-17 n+12)\end{array}$ \\
\hline
\end{tabular}

TABLE 11

Single Rational Function $o_{n}{ }^{(v)}: p_{n}{ }^{(0)}$

$\mathbf{A}^{\prime}$ : XXV, XXVII;

$B^{\prime}$ : XXV, XLII;

$C^{\prime}$ : I, II, XXVII, XXVIII

$\mathrm{U}^{\prime}$ : XXIX, XXXI, XXXII, XXVII, XXVIII;

$E^{\prime}$ : XXIX, XXXI. XXXII, YXXV, XXVII;

$F^{\prime}:$ XXIX, XXXV, XLII;

$\mathrm{G}^{\prime}:$ I, II, XXVIII, XLII;

$\mathrm{H}^{\prime}:$ I, II, XXVIII, XLV;

I': I, II, XLVII;

\begin{tabular}{|c|c|c|c|c|c|}
\hline & Multiplications & Divisions & Additions & Subtractions & Adds. + Subs. \\
\hline $\begin{array}{l}\mathbf{A}^{\prime} \\
\mathbf{B}^{\prime} \\
\mathbf{C}^{\prime} \\
\mathbf{D}^{\prime} \\
\mathbf{E}^{\prime} \\
\mathbf{F}^{\prime} \\
\mathbf{C}^{\prime} \\
\mathbf{H}^{\prime}\end{array}$ & $\begin{array}{l}3 n^{2}-5 n+2 \\
\frac{1}{2}\left(5 n^{2}-7 n+2\right) \\
3 n^{2}-5 n+2 \\
\frac{1}{6}\left(n^{3}+18 n^{2}\right. \\
\quad-19 n+12) \\
\frac{1}{3}\left(n^{3}+6 n^{2}-4 n\right. \\
\frac{1}{5}\left(2 n^{3}+9 n^{2}+3\right) \\
\frac{1}{2}\left(5 n^{2}-7 n+2\right) \\
\frac{1}{3}\left(n^{3}+6 n^{2}\right. \\
2 n^{2}-2 n\end{array}$ & $\begin{array}{l}2 n-1 \\
2 n-1 \\
n^{2}+1 \\
\frac{1}{2}\left(n^{2}+3 n\right) \\
\frac{1}{2}\left(n^{2}+3 n-2\right) \\
\frac{1}{2}\left(n^{2}+n\right) \\
n^{2}+1 \\
n^{2}+1 \\
n^{2}\end{array}$ & $\begin{array}{l}n^{2}-1 \\
3\left(n^{2}-n\right) \\
n^{2}-1 \\
2 n-2 \\
n-1 \\
\frac{1}{2}\left(n^{2}-n\right) \\
3\left(n^{2}-n\right) \\
\frac{1}{6}\left(n^{3}+6 n^{2}\right. \\
\quad-13 n+6) \\
\frac{1}{2}\left(3 n^{2}-7 n+4\right)\end{array}$ & $\begin{array}{l}3 n^{2}-6 n+3 \\
2 n^{2}-3 n+1 \\
3 n^{2}-6 n+3 \\
\frac{n^{2}}{6}\left(n^{3}+15 n^{2}\right. \\
\quad-22 n+12) \\
\frac{1}{6}\left(2 n^{3}+9 n^{2}\right. \\
\quad-11 n+6) \\
\frac{1}{6}\left(2 n^{3}+3 n^{2}+n\right) \\
2 n^{2}-3 n+1 \\
\frac{1}{6}\left(n^{3}+12 n^{2}\right. \\
\quad-19 n+6) \\
\frac{1}{2}\left(3 n^{2}-n-2\right)\end{array}$ & $\begin{array}{l}4 n^{2}-6 n+2 \\
\frac{1}{2}\left(7 n^{2}-9 n+2\right. \\
4 n^{2}-6 n+2 \\
\frac{1}{6}\left(n^{3}+15 n^{2}\right. \\
\frac{1}{6}\left(2 n^{3}+9 n^{2}-10 n\right. \\
\frac{1}{3}\left(n^{3}+3 n^{2}-n\right) \\
\frac{1}{2}\left(7 n^{2}-9 n+2\right) \\
\frac{1}{3}\left(n^{3}+9 n^{2}+1\right. \\
3 n^{2}-4 n+1\end{array}$ \\
\hline
\end{tabular}


Diagonal of Rational Functions $0_{r}(0): p_{r}(0) \quad r=1, \cdots, n$

$\mathbf{A}^{\prime}$ :

$\mathbf{J}^{\prime}: \mathbf{X X V}, \mathbf{X L I I I}$;

$D^{\prime}:$

$\mathrm{E}^{\prime}:$ XXIX, XXXV, XLIII;

$L^{\prime}:$ I, II, XXXIII, XLIII;

$\mathbf{H}^{\prime}$ :

$M^{\prime}:$ I, II, XLVIII;

\begin{tabular}{|c|c|c|c|c|c|}
\hline & Multiplications & Divisions & Additions & Subtractions & Adds. + Subs. \\
\hline $\begin{array}{l}\mathbf{D}^{\prime} \\
\mathbf{E}^{\prime}\end{array}$ & $\begin{array}{l}3 n^{2}-5 n+2 \\
\frac{1}{6}\left(n^{3}+12 n^{2}\right. \\
-19 n+6) \\
\frac{1}{6}\left(n^{3}+18 n^{2}\right. \\
\quad-19 n+12) \\
\frac{1}{3}\left(n^{3}+6 n^{2}-4 n\right. \\
\frac{1}{3}\left(n^{3}+2 n^{2}+n\right) \\
\frac{1}{6}\left(n^{3}+12 n^{2}\right. \\
\frac{1}{3}\left(n^{3}+6 n^{2}+6\right) \\
\frac{1}{3}\left(n^{3}+3 n^{2}-4 n\right)\end{array}$ & $\begin{array}{l}2 n-1 \\
2 n-1 \\
\frac{1}{2}\left(n^{2}+3 n\right) \\
\frac{1}{2}\left(n^{2}+3 n-2\right) \\
\frac{1}{2}\left(n^{2}+n\right) \\
n^{2}+1 \\
n^{2}+1 \\
n^{2}\end{array}$ & $\begin{array}{l}n^{2}-1 \\
\frac{1}{6}\left(n^{2}+6 n^{2}\right. \\
2 n-7 n) \\
n-1 \\
\frac{1}{6}\left(n^{3}-n\right) \\
\frac{1}{6}\left(n^{3}+6 n^{2}\right. \\
\quad-13 n+6) \\
\frac{1}{6}\left(n^{3}+6 n^{2}\right. \\
\quad-13 n+6) \\
\frac{1}{6}\left(n^{2}+3 n^{2}\right. \\
\quad-16 n+12)\end{array}$ & $\begin{array}{l}3 n^{2}-6 n+3 \\
2 n^{2}-3 n+1 \\
\quad\left(n^{3}+15 n^{2}\right. \\
-22 n+12) \\
\frac{1}{6}\left(2 n^{3}+9 n^{2}\right. \\
-11 n+6) \\
\frac{1}{6}\left(2 n^{3}+3 n^{2}+n\right) \\
2 n^{2}-3 n+1 \\
\frac{1}{6}\left(n^{3}+12 n^{2}\right. \\
\frac{19 n+6)}{6}\left(n^{3}+9 n^{2}\right. \\
-10 n)\end{array}$ & $\begin{array}{l}4 n^{2}-6 n+2 \\
\frac{1}{6}\left(n^{3}+18 n^{2}\right. \\
-25 n+6) \\
\frac{1}{2}\left(n^{3}+15 n^{2}\right. \\
-10 n) \\
\frac{1}{6}\left(2 n^{3}+9 n^{2}\right. \\
\frac{1}{3}\left(n^{3}+n^{2}\right) \\
\frac{1}{(}\left(n^{3}+18 n^{2}\right. \\
-31 n+12) \\
\frac{1}{3}\left(n^{3}+9 n^{2}\right. \\
\frac{16 n+6)}{3}\left(n^{3}+6 n^{2}\right. \\
-13 n+6)\end{array}$ \\
\hline
\end{tabular}

TABLE 13

Complete Array of Rational Functions $r_{r}{ }^{(m)}: p_{r}(m) \quad r=0, \cdots, n ; m=0, \cdots, 2 n-2 r$

$\mathrm{N}^{\prime}$ : XXVI, XXXVII, XXXIX;

$0^{\prime}$ : XXVI, Xixvil, XI, XIII;

$P^{\prime}:$ I, XXXVII, NXXIX;

$Q^{\prime}:$ I, XXIVIII, XI, XI,

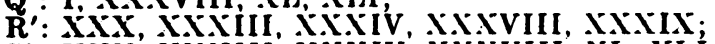

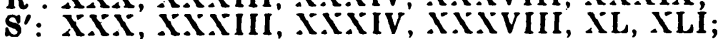

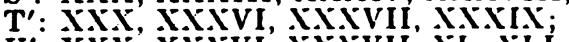

U': XXx, xixvi, XxiviI, XI, XII;

V': XXX', Xitvi, Xiliv;

$\mathrm{W}^{\prime}$ : XXX, XXXVI, XLVI;

$\mathrm{X}^{\prime}:$ I, II, XLIX;

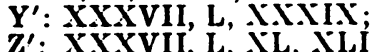

\begin{tabular}{|c|c|c|c|c|c|}
\hline & Multiplications & Divisions & Additions & Subtractions & Adds. + Subs. \\
\hline $\mathbf{N}^{\prime}$ & $\ln$ & $2 n^{2}$ & $\frac{1}{3}(2 n$ & $\frac{1}{f}\left(11 n^{3}-12\right.$ & $\frac{1}{2}\left(5 n^{3}-6 n^{2}+n\right)$ \\
\hline $\mathbf{O}^{\prime}$ & $\begin{array}{c}+13 n-6) \\
1\left(n^{4}+9 n^{3}-19 n^{2}\right.\end{array}$ & $2 n^{2}-2 n+1$ & $\frac{1}{3}\left(n^{4}+7 n^{3}\right.$ & $\frac{1}{6}\left(10 n^{3}-15 n^{t}\right.$ & $f\left(n^{4}+17\right.$ \\
\hline $\mathbf{P}^{\prime}$ & $\begin{array}{c}+15 n-6) \\
15 n^{3}+3 n^{2}\end{array}$ & & $\begin{array}{l}\left.-10 n^{2}+2 n\right) \\
n^{2}-3 n+2\end{array}$ & $\frac{1}{6}\left(5 n^{3}+3 n^{2}-8 n\right)$ & $\begin{array}{c}\left.-25 n^{2}+7 n\right) \\
t\left(5 n^{2}+9 n^{2}\right.\end{array}$ \\
\hline $\mathbf{Q}^{\prime}$ & $\left(n^{4}+7 n^{3} n+6\right)$ & $n^{2}$ & $1\left(n^{4}\right.$ & $\frac{2}{\frac{2}{3}}\left(n^{3}-n\right)$ & $\frac{1}{1}\left(n^{4}+7 n^{3}+12\right)$ \\
\hline & $\begin{array}{l}-4 n^{2}-10 n \\
+6)\end{array}$ & & $\begin{array}{l}+2 n^{2}-18 n \\
+12)\end{array}$ & & $\begin{array}{l}+2 n^{2}-22 n \\
+12)\end{array}$ \\
\hline $\mathbf{R}^{\prime}$ & $\begin{array}{l}1^{\frac{1}{2}}\left(n^{4}+18 n^{3}\right. \\
\left.+5 n^{2}\right)\end{array}$ & $\begin{array}{l}8\left(2 n^{3}+15 n^{2}\right. \\
-17 n+6)\end{array}$ & & $\begin{array}{l}1:\left(n^{4}+14 n^{3}\right. \\
\quad+23 n^{2}-50 n \\
+.0+)\end{array}$ & $\begin{array}{l}\frac{1}{12}\left(n^{4}+14 n^{3}\right. \\
\quad+23 n^{2}-50 n \\
\quad+2+4)\end{array}$ \\
\hline $\mathbf{S}^{\prime}$ & $\begin{array}{l}13 n^{4}+22 n^{3} \\
\left.-9 n^{2}+8 n\right)\end{array}$ & $\begin{array}{c}3\left(2 n^{3}+15 n^{2}\right. \\
-17 n\end{array}$ & $\begin{array}{c}\frac{1}{6}\left(n^{4}+3 n^{3}\right. \\
\left.-4 n^{2}\right)\end{array}$ & $\begin{array}{l}1=\left(n^{4}+12 n^{3}\right. \\
+5 n^{2}-18 n\end{array}$ & $\begin{array}{l}\frac{1}{2}\left(3 n^{4}+18 n^{3}\right. \\
-3 n^{2}-18 n\end{array}$ \\
\hline $\mathbf{T}^{\prime}$ & $\begin{array}{r}f\left(n^{4}+7 n^{3}+5 n^{2}\right. \\
-n)\end{array}$ & $\begin{array}{r}t\left(2 n^{3}+3 n^{2}\right. \\
+n+12)\end{array}$ & & $\begin{array}{l}1\left(n^{4}+5 n^{3}\right. \\
\quad+8 n^{2}-14 n \\
\quad+6)\end{array}$ & $\begin{array}{l}t\left(n^{4}+5 n^{3}\right. \\
\quad+8 n^{2}-14 n \\
\quad+6)\end{array}$ \\
\hline
\end{tabular}


TABLE 13-Continued

\begin{tabular}{|c|c|c|c|c|c|}
\hline & Multiplications & Divisions & Additions & Subtractions & Adds. + Subs. \\
\hline $\mathbf{U}^{\prime}$ & $\begin{array}{l}\frac{1}{6}\left(2 n^{4}+9 n^{3}\right. \\
\left.\quad-2 n^{2}+3 n\right)\end{array}$ & $\begin{array}{l}\frac{1}{6}\left(2 n^{2}+3 n^{2}\right. \\
\quad+n+12)\end{array}$ & $\begin{array}{r}\frac{1}{6}\left(n^{4}+3 n^{3}\right. \\
\left.-4 n^{2}\right)\end{array}$ & $\begin{array}{l}\frac{1}{6}\left(n^{4}+7 n^{3}+5 n^{2}\right. \\
-13 n+6)\end{array}$ & $\begin{array}{l}6\left(2 n^{4}+10 n^{2}\right. \\
\quad+n^{2}-13 n \\
+6)\end{array}$ \\
\hline $\begin{array}{l}\mathbf{V}^{\prime} \\
\mathbf{W}^{\prime}\end{array}$ & $\begin{array}{l}\frac{1}{3}\left(2 n^{4}+5 n^{3}\right. \\
\left.+4 n^{2}+n\right) \\
\frac{1}{3}\left(3 n^{4}+5 n^{3}\right. \\
\left.\quad+6 n^{2}-2 n\right)\end{array}$ & $\begin{array}{l}\frac{1}{6}\left(2 n^{3}+3 n^{2}\right. \\
+n+12) \\
6\left(2 n^{3}+3 n^{2}\right. \\
\quad+n+12)\end{array}$ & $\begin{array}{l}\frac{1}{6}\left(n^{4}-3 n^{3}\right. \\
\left.+2 n^{2}\right) \\
t_{2}^{2}\left(3 n^{4}+2 n^{3}\right. \\
\quad-9 n^{2}+16 n\end{array}$ & $\begin{array}{l}3\left(n^{4}+2 n^{3}\right. \\
\left.\quad+2 n^{2}+n\right) \\
\frac{1}{12}\left(5 n^{4}+6 n^{3}\right. \\
\left.\quad+7 n^{2}-6 n\right)\end{array}$ & $\begin{array}{l}\quad\left(2 n^{4}-n^{3}\right. \\
\left.+4 n^{2}+n\right) \\
\frac{2}{12}\left(5 n^{4}+6 n^{3}\right. \\
\left.+n^{2}+6 n-6\right)\end{array}$ \\
\hline $\mathbf{X}^{\prime}$ & $\frac{1}{6}\left(n^{4}+5 n^{2}-6 n\right)$ & $n^{2}$ & $\begin{array}{l}1 / 12) \\
\quad+35 n^{2}-8 n^{3} \\
\quad+36) \\
\quad+34 n\end{array}$ & $\begin{array}{l}1_{2}\left(n^{4}+4 n^{3}\right. \\
\left.\quad+5 n^{2}-10 n\right)\end{array}$ & $\begin{array}{l}\frac{1}{2}\left(n^{4}-2 n^{3}\right. \\
\quad+20 n^{2}-37 n \\
\quad+18)\end{array}$ \\
\hline $\mathbf{Y}^{\prime}$ & $\begin{array}{l}\frac{1}{5}\left(3 n^{4}+14 n^{3}\right. \\
+15 n^{2}-56 n \\
+24)\end{array}$ & $2 n^{2}-n$ & $\begin{array}{l}\frac{1}{12}\left(n^{4}+4 n^{3}\right. \\
\left.\quad-7 n^{2}+2 n\right)\end{array}$ & $\begin{array}{l}\frac{1}{\left(n^{4}+9 n^{3}+8 n^{2}\right.} \\
-24 n+12)\end{array}$ & $\begin{array}{l}1 \frac{1}{2}\left(3 n^{4}+22 n^{3}\right. \\
-9 n^{2}-46 n \\
+24)\end{array}$ \\
\hline $\mathbf{Z}^{\prime}$ & $\begin{aligned} \frac{1}{12} & \left(5 n^{4}+18 n^{3}+n^{2}\right. \\
& -48 n+24)\end{aligned}$ & $2 n^{2}-n$ & $\begin{array}{l}i_{2}\left(3 n^{4}+10 n^{3}\right. \\
\left.-15 n^{2}+2 n\right)\end{array}$ & $\begin{array}{r}1\left(n^{4}+8 n^{3}-n^{2}\right. \\
-20 n+12)\end{array}$ & $\begin{array}{l}\frac{1}{2}\left(5 n^{4}+26 n^{3}\right. \\
\quad-17 n^{2}-38 n \\
\quad+24)\end{array}$ \\
\hline
\end{tabular}

It appears from Tables 8 to 10 that, in terms of computational effort alone, a single entry in the $E$-array for a fixed value of the argument is most economically computed by means of the non-linear recursion

$$
\begin{aligned}
D_{1}=\frac{\beta_{n-2}^{(0)}}{z-\alpha_{n-1}^{(0)}} \quad & D_{s-1}=\frac{a_{n-s}}{b_{n-s}+D_{s}} \\
& a_{n}=c_{0} ; a_{s}=\beta_{s-2}^{(0)} ; s=2, \cdots, n ; b_{s}=z-\alpha_{s-1}^{(0)} ; s=1, \cdots, n
\end{aligned}
$$

in conjunction with the $q-d$ algorithm

$$
q_{r}^{(m)}+e_{r}^{(m)}=q_{r}^{(m+1)}+e_{r+1}^{(m+1)} \quad q_{r+1}^{(m)} e_{r}^{(m)}=q_{r}^{(m+1)} e_{r}^{(m+1)}
$$

and the numerical formation of the quantities

$$
\alpha_{r}{ }^{(0)}=e_{r}{ }^{(0)}+q_{r+1}^{(0)} \quad \beta_{r-1}^{(0)}=e_{r}{ }^{(0)} q_{r}{ }^{(0)} \quad r=1, \cdots, n-1 ;
$$

that a diagonal of quantities $E_{r}{ }^{(0)}(z) \quad r=1, \cdots, n$ is best computed by use of the $q-d$ algorithm, the formation of the quantities (6.22), the twin recursions

$$
\begin{gathered}
p_{r+1}^{(0)}(z)=\left(z-\alpha_{r}{ }^{(0)} p_{r}{ }^{(0)}(z)-\beta_{r-1}^{(0)} p_{r-1}^{(0)}(z), \quad r=1, \cdots, n\right. \\
p_{0}{ }^{(0)}(z)=1, \quad p_{1}{ }^{(0)}(z)=z-q_{1}{ }^{(0)} \\
o_{r+1}^{(0)}(z)=\left(z-\alpha_{r}{ }^{(0)}\right) o_{r}{ }^{(0)}(z)-\beta_{r-1}^{(0)} o_{r-1}^{(0)}(z), \quad r=1, \cdots, n \\
o_{0}{ }^{(0)}(z)=0, \quad o_{1}{ }^{(0)}(z)=c_{0}
\end{gathered}
$$

and the $n$ divisions

$$
E_{r}{ }^{(0)}(z)=\frac{o_{r}{ }^{(0)}(z)}{p_{r}{ }^{(0)}(z)} \quad r=1, \cdots, n,
$$

while the complete $E$-array of numerical values is best computed by evaluating the partial sums $E_{0}{ }^{(m)}(z)=\sum_{s-0}^{m-1} c_{s} z^{-s-1} m=1, \cdots, 2 n$, the quantities $E_{1 / 2}^{(m)}(z)$ $=z^{m} c_{m}{ }^{-1}$ and applying the $\epsilon$-algorithm

$$
E_{r+1 / 2}^{(m)}(z)=E_{r-1 / 2}^{(m+1)}(z)=\frac{1}{E_{r}^{(m+1)}(z)-E_{r}^{(m)}(z)} \quad r=\frac{1}{2}, 1, \frac{s}{2}, \cdots
$$


Tables 11 to 13 show, again in terms of computational effort alone, that the single rational expression $E_{n}{ }^{(0)}(z)$ be best constructed by determining the quantities $\alpha_{r}^{(0)}, \beta_{r-1}^{(0)}$ by means of the relationships

$$
\begin{aligned}
I\left\{t^{r} p_{r}{ }^{(0)}(t)\right\}=c_{0} \beta_{0}{ }^{(0)} & \cdots \beta_{r-1}^{(0)} \\
& I\left\{t^{r+1} p_{0}{ }^{(0)}(t)\right\}=c_{0} \beta_{0}{ }^{(0)} \cdots \beta_{r-1}^{(0)}\left\{\alpha_{0}{ }^{(0)}+\cdots+\alpha_{r}{ }^{(0)}\right\}
\end{aligned}
$$

in conjunction with the recursion (6.23), and of the vector $0_{n}{ }^{(0)}$ from

$$
\mathrm{O}_{n}{ }^{(0)}=\mathrm{p}_{n}{ }^{(0)}\left[\mathrm{C}_{n-1}^{(0)}\right]_{n+1, n}
$$

that the sequence of rational expressions $E_{r}{ }^{(0)}(z) \quad r=1, \cdots, n$ is best constructed by use of relationships ( 6.27$)$, in conjunction with the recursion $(6.23)$, determining the polynomials $o_{r}{ }^{(0)}(z)$ by means of the second recursion $(6.24)$; while the complete $E$-array of rational expressions is most efficiently constructed by use of the $q-d$ algorithm (6.22), in conjunction with the relationships

$$
\begin{gathered}
p_{r}{ }^{(m)}(z)=z p_{r-1}^{(m+1)}(z)-q_{r}{ }^{(m)} p_{r-1}^{(m)}(z) \quad p_{1}{ }^{(m)}(z)=z-q_{1}{ }^{(m)} \\
r_{r}^{(m)}(z)=z r_{r-1}^{(m+1)}(z)-q_{r}{ }^{(m)} r_{r-1}^{(m)}(z) \quad r_{0}{ }^{(m)}(z)=\sum_{s=0}^{m-1} c_{z} z^{-s-1}, \quad r_{0}{ }^{(0)}(z)=0
\end{gathered}
$$

Many electronic digital computers are equipped with highly flexible interpretive codes which are designed for carrying out standard matrix operations upon arrays of numbers. Many of the methods described may be susceptible to the use of such a code, and this consideration may cause the preceding judgements to be modified. The most suitable method is then largely determined by the exigencies of the particular code used. (This explains the presence, in the inquiry, of such methods as $E, H$, and $L$ which would otherwise be rejected outright.) The time taken for the operation of such a program may be estimated from the data given in the table, and a knowledge of the threshold time of each instruction.

Table 7 does not contain data relating to the direct solution of the equations

$$
\begin{aligned}
& k_{n, n}^{(m)} c_{0}=i_{n, m+n-1}^{(m)} \\
& k_{n, n-1}^{(m)} c_{0}+k_{n, n}^{(m)} c_{1}=i_{n, m+n-2}^{(m)}
\end{aligned}
$$

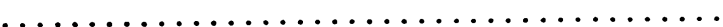

$$
\begin{aligned}
& k_{n, 0}^{(m)} c_{0}+k_{n, 1}^{(m)} c_{1}+\cdots+k_{n, n}^{(m)} c_{n}=i_{n, m-1}^{(m)} \\
& k_{n, 0}^{(m)} c_{1}+k_{n, 1}^{(m)} c_{2}+\cdots+k_{n n}^{(m)} c_{n+1}=i_{n, m-2}^{(m)}
\end{aligned}
$$

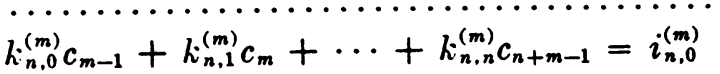

$$
\begin{aligned}
& k_{n, 0}^{(m)} c_{m}+k_{n, 1}^{(m)} c_{m+1}+\cdots+k_{n, n}^{(m)} c_{n+m}=0
\end{aligned}
$$

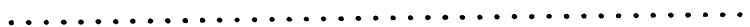

$$
\begin{aligned}
& k_{n, 0}^{(m)} c_{n+m-1}+k_{n, 1}^{(m)} c_{n+m}+\cdots+k_{n, n}^{(m)} c_{2 n+m-1}=0 \\
& k_{n, n}^{(m)}=1 \text {; }
\end{aligned}
$$

the details, of course, depend upon the method chosen for the solution of the equations. The solution of the set (6.31) is equivalent to the Gauss-Banachiervicz decomposition of the matrix

$$
\mathbf{C}_{n}{ }^{(m)}=\mathbf{H}_{n}{ }^{(m)} \mathbf{D}_{n}{ }^{(m)} \mathbf{H}_{n}{ }^{(m) T}
$$


the inversion of the matrix $H_{n}{ }^{(m)}$ to give $\mathbf{K}_{n}{ }^{(m)}$

$$
\mathbf{H}_{n}{ }^{(m)} \mathbf{K}_{n}{ }^{(m)}=I
$$

and the determination of the vector $r_{n}{ }^{(m)}$ from

$$
r_{n}{ }^{(m)}=p_{n}{ }^{(m)}\left[C_{m+n-1}^{(0)}\right]_{n+1, m+n} \text {. }
$$

This method (method $V^{\prime}$ above), if the steps involved are already incorporated in the interpretive code, is probably the one requiring the fewest instructions, and doubtless that consuming the most machine time.

(It may at first seem a little curious that the set of equations (6.31) does not involve the quantity $c_{m+2 n}$, whereas the matrix $\mathbf{C}_{n}{ }^{(m)}$ does. The quantity $c_{m+2 n}$, however, only enters into the determination of $\mathbf{H}_{n}{ }^{(m)}$ as a scaling factor of the last row, and this is removed by imposing the condition that the diagonal elements of $\mathbf{H}_{n}{ }^{(m)}$ are all unity. The quantity $c_{m+2 n}$ does not therefore enter into the determination of the matrix $\mathbf{K}_{n}{ }^{(m)}$.)

Apart from the considerations mentioned above, only those methods are described which might genuinely be suggested as the most economical. Many of the conclusions which may be deduced from a survey of Tables 8 to 13 might also have been arrived at after a preliminary discussion of the steps listed as I) to LVIII) but this discussion would have been so beset by qualification and modification that the most effective method of comparison was thought to be the straight forward enumeration of all feasible possibilities.

7. Checking and Correction of the Approximations. Since all the methods described in the last section are capable of producing the same final result, the computations produced by the use of one may be checked by application of any of the others, supplemented if necessary, by use of any of the theoretical results given in the text. In particular, attention is drawn to the strength of the use of the system of equations (6.31) as a final check.

Many of the methods entailed the computation of a sequence of polynomials: $p_{r}{ }^{(m)}(z) \quad r=0,1, \cdots$. The computation of the coefficients of these polynomials may be checked and corrected by a direct appeal to the orthogonality properties. Assuming the vectors $p_{s}{ }^{(m)} \quad s=0,1, \cdots, r-1$, to have been correctly computed, a corrected version $\mathbf{p}_{r_{\text {eorr. }}}^{(m)}$ of the vector $\mathbf{p}_{r}{ }^{(m)}$ may be produced from the formula

$$
\mathbf{p}_{r_{\text {oorr }}}^{(m)}=\mathbf{p}_{r}{ }^{(m)}-\sum_{s=0}^{r-1} \gamma_{r, s} \mathbf{p}_{s}{ }^{(m)}
$$

where

$$
\gamma_{r, e}=\mathbf{p}_{r}{ }^{(m)} \mathbf{c}_{r}{ }^{(m)}\left(\mathbf{p}_{s}{ }^{(m)}, 0, \cdots, 0\right)_{r+1}
$$

If the computation of the vector $p_{r}{ }^{(m)}$ has been performed without error, then it will transpire that

$$
\gamma_{r, s}=0 \quad s=0,1, \cdots, r-1 .
$$

The corrected vector $p_{r}{ }^{(m)}$ may now be used to check to computation of the vector $\mathbf{p}_{r+1}^{(m)}$ and so on.

Readers familiar with the computational finesses of Lanczos' [22] biortho- 
gonalization algorithm for determining the characteristic polynomial of a matrix will readily perceive that the use of equations (7.1) and (7.2) is a straightforward adaptation of a similar technique which relates to the use of this algorithm. Details of connections between this algorithm, the $q-d$ algorithm and certain others, which are significant in a theoretical context, are to be found in [10].

8. Acknowledgements. The author wishes to express his gratitude to E. L. Albasiny who made a number of useful criticisms of the paper. The above work was carried out partly when the author was a member of the Mathematisches Institut of the Technische Hochschule in München, and partly when he was a member of the Institut für Angewandte Mathematik in the University of Mainz; he is grateful to the deutsche Forschungsgemeinschaft for providing him with research grants which enabled it to be carried out.

Mathematisch Centrum

2e Boerhaavestraat 49

Amsterdam, Netherlands

1. H. PaDE, "Sur la Représentution Aprochée d'une Fonction par des Fractions Rationelles," Ann. Éc. Norm. Sup., v. 3, 1892, p. 9.

2. (). Perron, Die Lehre von den Kettenbrüchen, Chelsea, New York, 1950.

3. H. WALI, Analytic Theory of Continued Fractions, Van Nostrand, New York, 1948. 1953.

4. A. ERdei,yi, wT Al., Higher Transcendental Functions, v. 1, McGraw-Hill, New York,

5. T. J. S'Tieltjes, "Sur la Réduction en Fraction Continue d'une Série Précédant Suivant les Puissances I Descendants d'une Variable," Ann. Fac. Sci. Toulouse, v. 3, 1889, p. 1. 278.

6. H. Rutishauser, "Verallgemeinerung der Kettenbrüche," ZAMM, v. 38, 1958, p.

7. T. J. Streltses, “Récherches sur les Fractions Continues," Ann. Fac. Sci. Toulouse, v. 8,1894 , p. $1-122$.

8. A. C. AitKkin, “( )n Bernoulli's numerical solution of algebraic equations," Roy. Soc., Edin., Proc., v. 46, 1925-26, p. 289-305.

9. F. Stiefel, "Zur Berechnung höhere Eigenwerte Symmetrischer Operatoren mit Hilfe der Schwarzen Konst anten," Z.1MII, v. 33, 1953, p. 260-262.

10. H. Rutrsha user; Der Quotienten-Diferenzen-Algorithmus, Birkhauser Verlag, Basel, Switzerland, 1957.

11. P. HenkIC!. "'The (quotient difference algorithm," NBS Applied Mathematics Series, No. 49, 1958, p. $23-46$.

12. F. L. BAUER, "The g-algorithm," (to appear).

13. R. J. Schatit, "( nn the numerical solution of linear simultaneous equations by an iterative method," Phil. Mag., Ser. 7, v. 32, 1941, p. 369.

14. 1). SHANks, "Non-linear transformations of divergent and slowly convergent sequences," Jn. Wath. Physs., v. 34. 1955, p. 1-42. p. 91 .

15. P. WYNN, "On a device for computing the $e_{m}\left(S_{n}\right)$ transformation." $M T A C, v .10,1956$,

16. J. BARKLEY Rosier, "Theory and application of $\int_{0}^{x} e^{-x^{2}} d x$ and

$$
\int_{0}^{2} e^{-p^{2} y^{2}} d y \int_{0}^{y} e^{-x^{2}} d x,
$$

ORSD 5861, Mapleton House, Brooklyn, New York, 1948.

17. G. Frobenics. "Relationen zwischen den Näherungsbruchen von Potenzreihen," Jn. für Math., v. 90 , 1381 , p. 6.

18. J. H. MithLER, "On the application of continued fractions to the evaluation of certain integrals with specitic reference to the incomplete beta function," Biometrika, 1920, $22,21$.

19. P. S. LAPlaCe, Traite de Mécanique Céleste, Paris, 1802, 3 livre 10, p. 255.

20. British Association for the Advancement of Science, Mathematical Tables, v. 7, Cambridge University Press, 1939.

21. YUdell L. Like, "The Padé table and the r-method," Jn. Math. Phys., v. 37, 1958, p. 110.

22. C. Lanczos, "An iteration method for the solution of the eigenvalue problem of linear differential and integral operators," Jn. Res., NBS, v. 45, 1950, p. 255. 